\title{
Synthesis and Antioxidant and Antimicrobial Properties of $\beta$-Hydroxy Sulfides, Sulfoxides, and Sulfones Derived from Cardanol and Glycerol Derivatives
}

\author{
Suély Copini, ${ }^{a, b}$ Ana C. Micheletti, ${ }^{\circledR a}$ Dênis P. de Lima, ${ }^{\circledR a}$ Roberto S. Gomes, ${ }^{\circledR a, c}$ \\ Alisson Mezad ${ }^{d}$ and Adilson Beatriz ${ }^{\circledR *, a}$ \\ aInstituto de Química, Universidade Federal de Mato Grosso do Sul, \\ Av. Senador Filinto Muller, 1555, 79074-460 Campo Grande-MS, Brazil \\ ${ }^{b}$ Instituto Federal de Mato Grosso do Sul, Campus Corumbá, \\ Rua Pedro de Medeiros, s/n, 79310-110 Corumbá-MS, Brazil \\ 'Department of Pharmaceutical Sciences, North Dakota State University, \\ Fargo, ND, USA \\ ${ }^{d}$ Centro Universitário Anhanguera de Campo Grande, \\ Av. Gury Marques, 3203, 79060-000 Campo Grande-MS, Brazil
}

\begin{abstract}
Natural and synthetic sulfur-bearing organic compounds have many applications in medicinal chemistry. This article reports the preparation of amphiphilic $\beta$-hydroxy sulfides, sulfoxides, and sulfones derived from cardanol and glycerol. Thiolysis of cardanol epoxide $\mathbf{3}$ with various thiols $(\mathbf{4 a}-\mathbf{4 j})$ in ethanol or water yielded the corresponding $\beta$-hydroxy sulfides $\mathbf{5 a - 5 j}$ (61-95\%). Treatment with $30 \% \mathrm{H}_{2} \mathrm{O}_{2}$ in acetic acid at ambient temperature completely converted these products into $\beta$-hydroxy sulfoxides $\mathbf{6 a - 6 j}$ and sulfones $\mathbf{7 a}, \mathbf{7} \mathbf{c}-\mathbf{7 f}, \mathbf{7 h}-\mathbf{7} \mathbf{j}$. The synthesized compounds were characterized by ${ }^{1} \mathrm{H}$ nuclear magnetic resonance (NMR), ${ }^{13} \mathrm{C}$ NMR, high-resolution mass spectroscopy (HRMS) and performed the in vitro evaluation antimicrobial activities against standard strains of Staphylococcus aureus and Escherichia coli. Compounds $\mathbf{5 a}, \mathbf{5 d}-\mathbf{5 f}$, and $\mathbf{5 h}-\mathbf{5} \mathbf{j}$ proved moderately active against $S$. aureus. None of the compounds were active against $E$. coli. $\beta$-Hydroxy-sulfides $\mathbf{5 a - 5 j}$ were also evaluated for antioxidant properties but failed to exhibit significant activity.
\end{abstract}

Keywords: CNSL, cardanol, hydroxy sulfides, epoxide ring opening, antibacterial activity

\section{Introduction}

Sulfur-bearing organic compounds are ubiquitous in nature. Because sulfur is, after chlorine, the most abundant element in sea water, organosulfur compounds are typically isolated from marine organisms. ${ }^{1,2}$ In the molecular architecture of these natural compounds, sulfur can be a component of various functional groups, exhibiting differing states of oxidation, as in thiols, sulfides (acyclic or heterocyclic), disulfides, sulfoxides, and sulfonates. ${ }^{2,3}$ Antioxidant, antimicrobial, anti-inflammatory, antitumor, anti-HIV, and calcium-channel-antagonist activities make natural and synthetic organic compounds containing sulfur useful for numerous applications in medicinal chemistry. ${ }^{4}$ $\beta$-Hydroxy sulfides are constituents of several biologically

*e-mail: adilson.beatriz@ufms.br relevant synthetic materials. ${ }^{2}$ Figure 1 shows examples of natural and synthetic compounds of clinical value that incorporate $\beta$-hydroxy sulfides. Leukotriene E4 (LTE4), isolated from mast cells and extensively investigated for allergy and asthma, is a member of a family of leukocyte-generated eicosanoids containing $\beta$-hydroxy sulfides. ${ }^{5}$ Pteriatoxin A, isolated from a Japanese mollusk, has proven an extremely potent neurotoxin to mice. ${ }^{6}$ Cyclothiocurvularin is a fungal product isolated from Penicillium sp. ${ }^{7}$ Diltiazem and naltiazem are synthetic $\beta$-hydroxy sulfides used as calcium channel blockers in the treatment of hypertension, angina pectoris, and some types of cardiac arrhythmia. ${ }^{8}$

In line with our interest to explore the Brazilian biomass for substances of special structural and chemical interest, our research group has been investigating the potential of cardanol (1) and glycerol (2) to yield hybrid compounds 


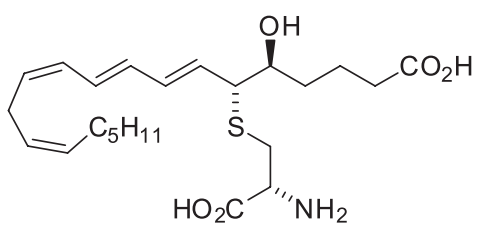

leukotriene E4 (LTE4)<smiles>CCCCCCCOC(=O)O[C@H](C)CCC[C@H]1SC[C@](O)(C(=O)OCc2ccccc2)[C@H]1C(=O)O</smiles>

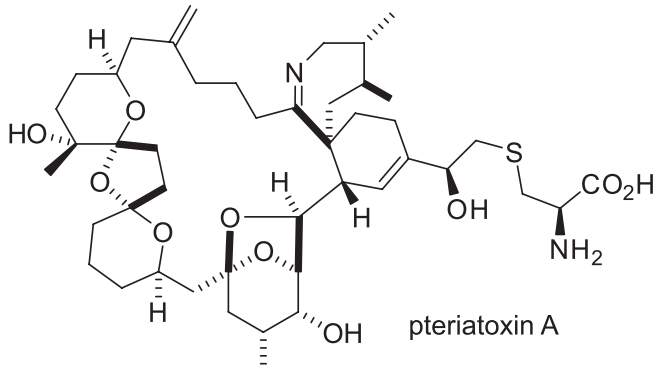<smiles>COc1ccc([C@H]2Sc3ccccc3N(CCN(C)C)C(=O)[C@H]2OC(C)=O)cc1</smiles>

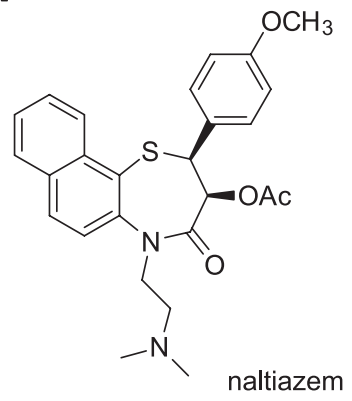

Figure 1. Examples of $\beta$-hydroxy sulfides found in natural (leukotriene E4, pteriatoxin A, cyclothiocurvularin) and synthesized products (diltiazem and naltiazem).

for biological and technological applications. Cardanol is the main phenolic component in technical-grade cashew nutshell liquid (CNSL). Anacardic acid, abundant in these shells, undergoes decarboxylation when the nuts are roasted for human consumption, yielding cardanols with a range of unsaturated alkyl chains ${ }^{9}$ (Scheme 1).

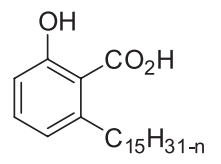

anacardic acid

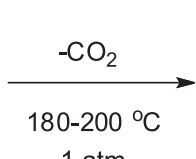

$1 \mathrm{~atm}$

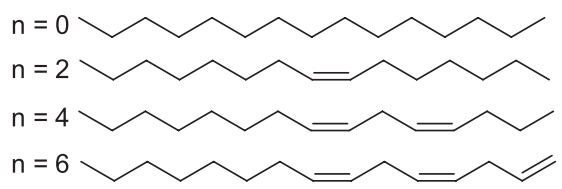

Scheme 1. Decarboxylation of anacardic acid.

The scientific community and the industrial sector hold a consensus that glycerol availability is critical for largescale biodiesel production, hence the growing interest in alternative sources of glycerol, whether as crude material or as high-added-value derivatives. ${ }^{10}$

We prepared cardanol epoxide 3 (Figure 2) from saturated cardanol $(\mathrm{n}=0)$ and racemic epichlorohydrin (a glycerol derivative). The cardanol epoxide molecule is suitable for achieving oxirane ring opening upon attack by a range of nucleophiles, including amines, halogens, alcohols, and hydroxide ions. We recently reported the preparation of fluorescent lipophilic compounds for use as fuel markers (4-6), ${ }^{11}$ lipophilic antimicrobials (7), ${ }^{12}$ and potent larvicides (10) against Aedes aegypti larvae ${ }^{13}$ (Figure 2).

The great versatility of cardanol epoxide has boosted our interest in obtaining amphiphilic $\beta$-hydroxy sulfides through ring opening with thiols and subsequent oxidation of sulfides to sulfoxides and corresponding sulfones.

$\beta$-Hydroxy sulfides can be generated by thiolyzing epoxides using catalysts such as rongalite in the presence of potassium carbonate in dimethylformamide (DMF) ${ }^{14}$ or amberlyst- 15 in toluene. ${ }^{15}$ Other methods have been developed ${ }^{16}$ using Lewis or Brønsted acids, such as

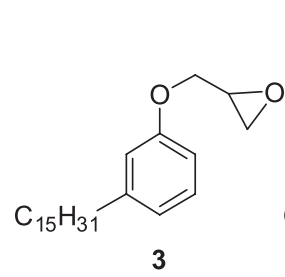

3<smiles>CCCCCc1cccc(OCC(O)Cn2cc(-c3ccc(N)cc3)nn2)c1</smiles>

4, ortho $\left(\mathrm{NH}_{2}\right)$

5, meta $\left(\mathrm{NH}_{2}\right)$

6, para $\left(\mathrm{NH}_{2}\right)$<smiles>[R]NCC(O)COc1cccc([13CH3])c1</smiles>

7, R = aryl, alkyl<smiles>[X]CC(O)COc1cccc([AsH2])c1</smiles>

8, $\mathrm{X}=\mathrm{Cl}$

9, $\mathrm{X}=\mathrm{Br}$

$10, X=1$

Figure 2. Examples of cardanol and glycerol derivatives. 


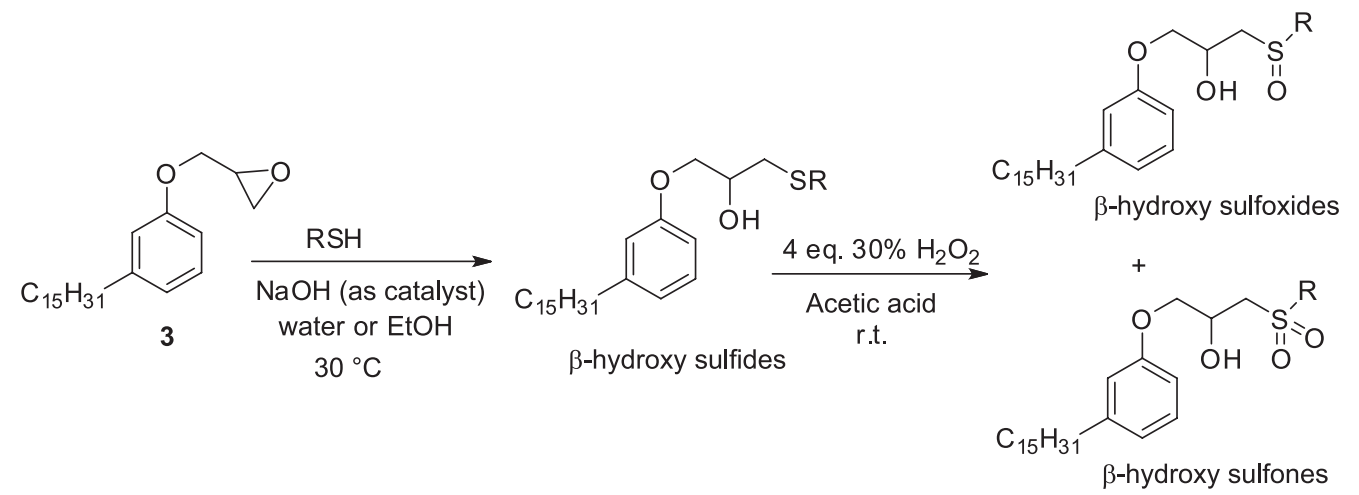

Scheme 2. Synthesis of $\beta$-hydroxy sulfides, sulfoxides, and sulfones derived from cardanol and glycerol.

$\mathrm{B}\left(\mathrm{C}_{6} \mathrm{~F}_{5}\right)_{3},{ }^{17} \mathrm{InCl}_{3},{ }^{18} \mathrm{ZnCl}_{2},{ }^{19} \mathrm{Ga}(\mathrm{OTf}){ }_{3},{ }^{20}$ and $\mathrm{HBF}_{4}-\mathrm{SiO}_{2} \cdot{ }^{21}$ Attempting to minimize the use of noxious organic solvents, some investigators ${ }^{22,23}$ have achieved epoxide thiolysis in water in the presence or absence of a base. Searching for an environmentally friendly process ${ }^{24}$ we chose to perform thiolysis in aqueous or ethanol media, with subsequent sulfide oxidation using $30 \% \mathrm{H}_{2} \mathrm{O}_{2}$ in glacial acetic acid (Scheme 2).

The fact that the lipophilic nature of some antibiotics has a significant effect on antibacterial activity ${ }^{12}$ furthered our group's interest in developing novel antimicrobials, prompting us to design novel, potentially bioactive molecules. The generated organosulfur compounds were evaluated for antimicrobial and antioxidant properties.

\section{Experimental}

\section{General methods}

All reagents and solvents used were commercially obtained (Merck, São Paulo, Brazil and/or Labsynth, São Paulo, Brazil) and used as purchased. The technical cashew (Anacardium occidentale L.) nutshell liquid (tCNSL) was donated by Kardol Ind. Química (Campo Grande, Brazil). Cardanol, the major constituent of tCNSL, was isolated according to the method described in patent BR 102014030002-3 A2. ${ }^{25}$ Catalytic hydrogenation was carried out in a Parr hydrogenation apparatus, applying our previously reported method. ${ }^{11}$ Thin layer chromatography (TLC) was performed on glass plates coated with silica gel 60 F254 (Merck, São Paulo, Brazil). The plates were visualized using UV radiation $(254 \mathrm{~nm})$, iodine, or both. Column chromatography was performed on Merck silica gel $(60 \times 120 \mathrm{mesh})$ in a glass column. ${ }^{1} \mathrm{H}$ and ${ }^{13} \mathrm{C}$ nuclear magnetic resonance (NMR) spectra were recorded on a Bruker Avance DPX-300 apparatus using tetramethylsilane (TMS) as the internal standard. Chemical shifts $(\delta)$ were recorded in ppm with respect to TMS, with coupling constants $(J)$ given in hertz. Multiplicity: br $=$ broad, $\mathrm{s}=$ singlet, $\mathrm{d}=$ doublet, $\mathrm{t}=$ triplet, $\mathrm{m}=$ multiplet. High-resolution mass spectroscopy (HRMS) was performed on a UFLC Shimadzu LC-20AD device equipped with a Bruker Daltonics IES-Q-QTOF-microTOF III detector operating in chemical-ionization positive-ion mode $(\mathrm{m} / \mathrm{z}, 120-1200)$.

\section{Experimental procedure}

\section{Synthesis of $\beta$-hydroxy sulfides $\mathbf{5 a}-\mathbf{5} \mathbf{j}$}

A $2 \mathrm{~mL}$ volume of $0.03 \mathrm{M}$ sodium hydroxide in ethanol was added to an ethanol solution $(2 \mathrm{~mL})$ of $1 \mathrm{mmol}$ cardanol epoxide $3^{11}$ and $1.0 \mathrm{mmol}$ thiol at ambient temperature and the mixture stirred at $30^{\circ} \mathrm{C}$ for $2 \mathrm{~h}$. The reaction was monitored by TLC, the resulting mixture allowed to cool to room temperature, and ethanol finally removed under vacuum. The remaining mixture was partitioned between water $(20 \mathrm{~mL})$ and ethyl acetate $(25 \mathrm{~mL})$, the organic layer separated, and the aqueous layer extracted with ethyl acetate $(2 \times 20 \mathrm{~mL})$ and dried over $\mathrm{MgSO}_{4}$. The solvent was removed under vacuum and the crude compound was purified by column chromatography using 20-50\% EtOAc in hexane as the eluent to yield the desired $\beta$-hydroxy sulfides $(\mathbf{5 a}-\mathbf{5 j})$.

${ }^{1} \mathrm{H}$ NMR, ${ }^{13} \mathrm{C}$ NMR, and MS data of isolated compounds

1-(3-Pentadecylphenoxy)-3-(phenylthio)propan-2-ol (5a)

Yield: 80\%; white solid; ${ }^{1} \mathrm{H}$ NMR $\left(300 \mathrm{MHz}, \mathrm{CDCl}_{3}\right.$ ) $\delta 0.80\left(\mathrm{t}, 3 \mathrm{H}, J 6.0 \mathrm{~Hz}, \mathrm{CH}_{3}\right), 1.18\left(\mathrm{~m}, 24 \mathrm{H}, 12 \mathrm{CH}_{2}\right), 1.51$ (m, $2 \mathrm{H}, \mathrm{CH}_{2}$ ), 2.31 (br, $\left.1 \mathrm{H}, \mathrm{OH}\right), 2.47\left(\mathrm{t}, 2 \mathrm{H}, J 7.7 \mathrm{~Hz}, \mathrm{CH}_{2}\right.$ ), 3.03-3.20 (m, 2H, $\left.\mathrm{CH}_{2}\right), 3.90-3.95\left(\mathrm{~m}, 2 \mathrm{H}, \mathrm{CH}_{2}\right), 4.00-4.04$ (m, 1H, CH), 6.59-6.62 (m, 2H, Ar-H), 6.69-6.72 (d, 1H, J 7.5 Hz, Ar-H), 7.06-7.13 (m, 2H, Ar-H), 7.16-7.22 (m, 2H, Ar-H), 7.31-7.34 (m, 2H, Ar-H); ${ }^{13} \mathrm{C}$ NMR (75 MHz, $\left.\mathrm{CDCl}_{3}\right) \delta 14.1,22.7,29.3,29.5,29.6,29.7,31.4,31.9$, $35.9,36.0,68.6,70.0,111.5,114.7,121.4,126.5,129.1$, 
129.2, 129.8, 135.1, 144.7, 158.3; HRMS (electrospray ionization (ESI)) $\mathrm{m} / z$, calcd. for $\mathrm{C}_{30} \mathrm{H}_{47} \mathrm{O}_{2} \mathrm{~S}^{+}[\mathrm{M}+\mathrm{H}]^{+}$: 471.3291, found: 471.3237 .

1-(3-Pentadecylphenoxy)-3-(p-tolylthio)propan-2-ol (5b)

Yield: $62 \%$; white solid; ${ }^{1} \mathrm{H}$ NMR $\left(300 \mathrm{MHz}, \mathrm{CDCl}_{3}\right.$ ) $\delta 0.85\left(\mathrm{t}, 3 \mathrm{H}, J 6.9 \mathrm{~Hz}, \mathrm{CH}_{3}\right), 1.24-1.29\left(\mathrm{~m}, 24 \mathrm{H}, 12 \mathrm{CH}_{2}\right)$, 1.55-1.62 (m, 2H, $\left.\mathrm{CH}_{2}\right), 2.30\left(\mathrm{br}, 3 \mathrm{H}, \mathrm{CH}_{3}\right), 2.51-2.57(\mathrm{t}$, $2 \mathrm{H}, J 7.6 \mathrm{~Hz}, \mathrm{CH}_{2}$ ), 2.69-2.74 (br, $\left.1 \mathrm{H}, \mathrm{OH}\right), 3.05-3.22$ (m, $\left.2 \mathrm{H}, \mathrm{CH}_{2}\right), 3.95-4.08\left(\mathrm{~m}, 3 \mathrm{H}, \mathrm{CH}_{2}\right.$ and $\left.\mathrm{CH}\right), 6.65-6.68$ (m, 2H, Ar-H), 6.77 (d, 1H, J 7.4 Hz, Ar-H), $7.08(\mathrm{~d}, 2 \mathrm{H}$, $J 7.9 \mathrm{~Hz}, \mathrm{Ar}-\mathrm{H}), 7.15$ (t, $1 \mathrm{H}, J 7.6 \mathrm{~Hz}, \mathrm{Ar}-\mathrm{H}), 7.29$ (d, 2H, $J$ 7.9 Hz, Ar-H); ${ }^{13} \mathrm{C}$ NMR (75 MHz, $\left.\mathrm{CDCl}_{3}\right) \delta 14.1,21.0$, 22.7, 29.3, 29.5, 29.6, 29.7, 31.4, 31.9, 35.9, 38.3, 68.5, 69.9, 111.5, 114.7, 121.4, 129.1, 129.9, 130.7, 136.9, 144.7, 158.4; HRMS (ESI) $\mathrm{m} / z$, calcd. for $\mathrm{C}_{31} \mathrm{H}_{49} \mathrm{O}_{2} \mathrm{~S}^{+}[\mathrm{M}+\mathrm{H}]^{+}$: 485.3448, found: 485.3468 .

1-((4-Fluorophenyl)thio)-3-(3-pentadecylphenoxy)propan2-ol (5c)

Yield: $80 \%$; white solid; ${ }^{1} \mathrm{H}$ NMR (300 $\mathrm{MHz}, \mathrm{CDCl}_{3}$ ) $\delta 0.79\left(\mathrm{t}, 3 \mathrm{H}, J 6.4 \mathrm{~Hz}, \mathrm{CH}_{3}\right), 1.17-1.22\left(\mathrm{~m}, 24 \mathrm{H}, 12 \mathrm{CH}_{2}\right)$, $1.45-1.55\left(\mathrm{~m}, 2 \mathrm{H}, \mathrm{CH}_{2}\right), 2.49\left(\mathrm{t}, 2 \mathrm{H}, J 7.7 \mathrm{~Hz}, \mathrm{CH}_{2}\right), 2.55$ (br, $1 \mathrm{H}, \mathrm{OH}), 2.96-3.12\left(\mathrm{~m}, 2 \mathrm{H}, \mathrm{CH}_{2}\right), 3.87-3.99(\mathrm{~m}, 3 \mathrm{H}$, $\mathrm{CH}_{2}$ and $\left.\mathrm{CH}\right), 6.57-6.60(\mathrm{~m}, 2 \mathrm{H}, \mathrm{Ar}-\mathrm{H}), 6.69(1 \mathrm{~d}, 1 \mathrm{H}$, $J 7.5 \mathrm{~Hz}, \mathrm{Ar}-\mathrm{H}), 6.87$ (t, $2 \mathrm{H}, J 8.7 \mathrm{~Hz}, \mathrm{Ar}-\mathrm{H}), 7.05(\mathrm{t}$, $1 \mathrm{H}, J 7.7 \mathrm{~Hz}, \mathrm{Ar}-\mathrm{H}), 7.30$ (dd, 2H, J 8.8, $5.2 \mathrm{~Hz}, \mathrm{Ar}-\mathrm{H}$ ); ${ }^{13} \mathrm{C}$ NMR $\left(75 \mathrm{MHz}, \mathrm{CDCl}_{3}\right) \delta 14.1,22.7,29.3,29.5,29.6$, 29.7, 31.3, 31.9, 35.9, 38.7, 68.6, 69.9, 111.4, 114.7, $116.0,116.3,121.4,129.1,130.0,130.1,132.7,132.8$, 144.7, 158.2, 160.4, 163.6; HRMS (ESI) $\mathrm{m} / \mathrm{z}$, calcd. for $\mathrm{C}_{30} \mathrm{H}_{46} \mathrm{FO}_{2} \mathrm{~S}^{+}[\mathrm{M}+\mathrm{H}]^{+}:$: 489.3197, found: 489.3189 .

1-((2-Chlorophenyl)thio)-3-(3-pentadecylphenoxy)propan2-ol (5d)

Yield: 90\%; white solid; ${ }^{1} \mathrm{H}$ NMR $\left(300 \mathrm{MHz}, \mathrm{CDCl}_{3}\right)$ $\delta 0.77\left(\mathrm{t}, 3 \mathrm{H}, J 6.4 \mathrm{~Hz}, \mathrm{CH}_{3}\right), 1.12-1.22\left(\mathrm{~m}, 24 \mathrm{H}, 12 \mathrm{CH}_{2}\right)$, 1.51-1.55 (m, 2H, $\left.\mathrm{CH}_{2}\right), 2.50\left(\mathrm{t}, 2 \mathrm{H}, J 7.7 \mathrm{~Hz}, \mathrm{CH}_{2}\right)$, 3.04-3.23 (m, 2H, $\left.\mathrm{CH}_{2}\right), 3.93-3.98\left(\mathrm{~m}, 2 \mathrm{H}, \mathrm{CH}_{2}\right), 4.00-4.08$ $(\mathrm{m}, 1 \mathrm{H}, \mathrm{CH}), 6.59-6.64(\mathrm{~m}, 2 \mathrm{H}, \mathrm{Ar}-\mathrm{H}), 6.70(\mathrm{~d}, 1 \mathrm{H}$, J 7.5 Hz, Ar-H), 7.00-7.15 (m, 3H, Ar-H), 7.26-7.33 (m, 2H, $\mathrm{Ar}-\mathrm{H}) ;{ }^{13} \mathrm{C} \mathrm{NMR}\left(75 \mathrm{MHz}, \mathrm{CDCl}_{3}\right) \delta 14.2,22.7,29.4,29.5$, 29.6, 29.7, 31.4, 31.9, 36.0, 36.5, 68.6, 70.0, 111.5, 114.8, $121.5,127.2$, 127.3, 129.3, 129.6, 129.9, 134.3, 134.6, 144.8, 158.3; HRMS (ESI) $m / z$, calcd. for $\mathrm{C}_{30} \mathrm{H}_{46} \mathrm{ClO}_{2} \mathrm{~S}^{+}$ $[\mathrm{M}+\mathrm{H}]^{+}:$505.2902, found: 505.2901.

1-((3-Chlorophenyl)thio)-3-(3-pentadecylphenoxy)propan2-ol (5e)

Yield: 65\%; white solid; ${ }^{1} \mathrm{H}$ NMR $\left(300 \mathrm{MHz}, \mathrm{CDCl}_{3}\right)$ $\delta 0.80\left(\mathrm{t}, 3 \mathrm{H}, J 6.4 \mathrm{~Hz}, \mathrm{CH}_{3}\right), 1.18-1.23\left(\mathrm{~m}, 24 \mathrm{H}, 12 \mathrm{CH}_{2}\right)$,
1.49-1.54 (m, 2H, $\left.\mathrm{CH}_{2}\right), 2.49\left(\mathrm{t}, 2 \mathrm{H}, J 7.7 \mathrm{~Hz}, \mathrm{CH}_{2}\right)$, 3.05-3.22 (m, 2H, $\left.\mathrm{CH}_{2}\right), 3.92-3.97\left(\mathrm{~m}, 2 \mathrm{H}, \mathrm{CH}_{2}\right), 4.01-4.08$ (m, 1H, CH), 6.60-6.64 (m, 2H, Ar-H), $6.72(\mathrm{~d}, 1 \mathrm{H}$, J 7.4 Hz, Ar-H), 7.09-7.14 (m, 3H, Ar-H), 7.17-7.18 (m, 1H, Ar-H), 7.30-7.31 (br, 1H, Ar-H); ${ }^{13} \mathrm{C}$ NMR (75 MHz, $\left.\mathrm{CDCl}_{3}\right) \delta 14.1,22.7,29.4,29.5,29.6,29.7,31.4,31.9$, 36.0, 37.1, 68.6, 69.8, 111.4, 114.7, 121.5, 126.5, 127.3, 128.8, 129.2, 130.0, 134.8, 137.5, 144.8, 158.2; HRMS (ESI) $m / z$, calcd. for $\mathrm{C}_{30} \mathrm{H}_{46} \mathrm{ClO}_{2} \mathrm{~S}^{+}[\mathrm{M}+\mathrm{H}]^{+}:$505.2902, found: 505.2901.

1-((4-Chlorophenyl)thio)-3-(3-pentadecylphenoxy)propan2-ol (5f)

Yield: $88 \%$; white solid; ${ }^{1} \mathrm{H}$ NMR $\left(300 \mathrm{MHz}, \mathrm{CDCl}_{3}\right)$ $\delta 0.80\left(\mathrm{t}, 3 \mathrm{H}, J 6.4 \mathrm{~Hz}, \mathrm{CH}_{3}\right), 1.18-1.23\left(\mathrm{~m}, 24 \mathrm{H}, 12 \mathrm{CH}_{2}\right)$, 1.49-1.54 (m, 2H, $\left.\mathrm{CH}_{2}\right), 2.49\left(\mathrm{t}, 2 \mathrm{H}, J 7.6 \mathrm{~Hz}, \mathrm{CH}_{2}\right)$, 3.02-3.29 (m, 2H, $\left.\mathrm{CH}_{2}\right), 3.90-4.03\left(\mathrm{~m}, 3 \mathrm{H}, \mathrm{CH}_{2}\right.$ and $\left.\mathrm{CH}\right)$, 6.59-6.62 (m, 2H, Ar-H), 6.71 (d, 1H, J 7.4 Hz, Ar-H), 7.10 (t, 1H, J 7.6 Hz, Ar-H), 7.16 (d, 2H, J 8.4 Hz, Ar-H), 7.26 (d, 2H, J 8.5 Hz, Ar-H); ${ }^{13} \mathrm{C}$ NMR (75 MHz, $\mathrm{CDCl}_{3}$ ) $\delta 14.1,22.7,29.3,29.5,29.6,29.7,31.4,31.9,35.9,37.7$, 68.6, 69.9, 111.4, 114.7, 121.5, 129.1, 131.0, 132.6, 133.7, 144.8, 158.2; HRMS (ESI) $\mathrm{m} / z$, calcd. for $\mathrm{C}_{30} \mathrm{H}_{46} \mathrm{BrO}_{2} \mathrm{~S}^{+}$ $[\mathrm{M}+\mathrm{H}]^{+}:$549.2396, found: 549.2394.

1-((2-Bromophenyl)thio)-3-(3-pentadecylphenoxy)propan2-ol (5g)

Yield: 73\%; white solid; ${ }^{1} \mathrm{H}$ NMR $\left(300 \mathrm{MHz}, \mathrm{CDCl}_{3}\right)$ $\delta 0.80\left(\mathrm{t}, 3 \mathrm{H}, J 6.8 \mathrm{~Hz}, \mathrm{CH}_{3}\right), 1.17-1.26\left(\mathrm{~m}, 22 \mathrm{H}, 11 \mathrm{CH}_{2}\right)$, 1.48-1.59 (m, 2H, $\left.\mathrm{CH}_{2}\right), 2.43(\mathrm{br}, 1 \mathrm{H}, \mathrm{OH}), 2.48(\mathrm{t}, 2 \mathrm{H}$, J 7.7 Hz, $\left.\mathrm{CH}_{2}\right), 3.05-3.23\left(\mathrm{~m}, 2 \mathrm{H}, \mathrm{CH}_{2}\right), 3.97-3.99(\mathrm{~m}, 2 \mathrm{H}$, $\mathrm{CH}_{2}$ ), 4.02-4.10 (m, 1H, CH), 6.60-6.65 (m, 2H, Ar-H), 6.71 (d, 1H, J 7.5 Hz, Ar-H), 6.92-6.98 (m, 1H, Ar-H), 7.06-7.18 (m, 2H, Ar-H), 7.29-7.32 (m, 1H, Ar-H), 7.45-7.48 (m, 1H, $\mathrm{Ar}-\mathrm{H}) ;{ }^{13} \mathrm{C} \mathrm{NMR}\left(75 \mathrm{MHz}, \mathrm{CDCl}_{3}\right) \delta 14.1,22.6,29.3,29.5$, 29.6, 29.7, 31.4, 31.9, 34.8, 35.9, 36.8, 68.5, 69.9, 111.5, $114.7,121.5,124.4,127.3,127.9,129.2,133.1,136.6$, 144.7, 158.2; HRMS (ESI) $m / z$, calcd. for $\mathrm{C}_{30} \mathrm{H}_{46} \mathrm{BrO}_{2} \mathrm{~S}^{+}$ $[\mathrm{M}+\mathrm{H}]^{+}:$549.2396, found: 549.2224.

1-((4-Bromophenyl)thio)-3-(3-pentadecylphenoxy)propan2-ol (5h)

Yield: 67\%; white solid; ${ }^{1} \mathrm{H}$ NMR $\left(300 \mathrm{MHz}, \mathrm{CDCl}_{3}\right.$ ) $\delta 0.80\left(\mathrm{t}, 3 \mathrm{H}, J 6.4 \mathrm{~Hz}, \mathrm{CH}_{3}\right), 1.18-1.23\left(\mathrm{~m}, 24 \mathrm{H}, 12 \mathrm{CH}_{2}\right)$, 1.49-1.54 (m, 2H, $\left.\mathrm{CH}_{2}\right), 2.22(\mathrm{br}, 1 \mathrm{H}, \mathrm{OH}), 2.49(\mathrm{t}, 2 \mathrm{H}$, J 7.7 Hz, $\left.\mathrm{CH}_{2}\right), 3.02-3.19\left(\mathrm{~m}, 2 \mathrm{H}, \mathrm{CH}_{2}\right), 3.93-3.96(\mathrm{~m}, 2 \mathrm{H}$, $\left.\mathrm{CH}_{2}\right), 3.98-4.03$ (m, 1H, CH), 6.59-6.62 (m, 2H, Ar-H), 6.72 (d, 1H, J 7.5 Hz, Ar-H), 7.08 (t, 1H, J 7.7 Hz, ArH), 7.17-7.19 (m, 2H, Ar-H), 7.29-7.33 (m, 2H, Ar-H); ${ }^{13} \mathrm{C} \mathrm{NMR}\left(75 \mathrm{MHz}, \mathrm{CDCl}_{3}\right) \delta 14.1,22.7,29.3,29.5,29.6$, 29.7, 31.4, 31.9, 35.9, 37.5, 68.6, 69.9, 111.4, 114.7, 120.4, 
121.5, 129.2, 131.2, 132.1, 134.5, 144.8, 158.2; HRMS (ESI) $\mathrm{m} / z$, calcd. for $\mathrm{C}_{30} \mathrm{H}_{46} \mathrm{BrO}_{2} \mathrm{~S}^{+}[\mathrm{M}+\mathrm{H}]^{+}:$549.2396, found: 549.2224 .

1-((4-Aminophenyl)thio)-3-(3-pentadecylphenoxy)propan2-ol (5i)

Yield: 61\%; light yellow oil; ${ }^{1} \mathrm{H}$ NMR $(300 \mathrm{MHz}$, $\left.\mathrm{CDCl}_{3}\right) \delta 0.81\left(\mathrm{t}, 3 \mathrm{H}, J 6.5 \mathrm{~Hz}, \mathrm{CH}_{3}\right), 1.18-1.22(\mathrm{~m}, 24 \mathrm{H}$, $\left.12 \mathrm{CH}_{2}\right), 1.49-1.54\left(\mathrm{~m}, 2 \mathrm{H}, \mathrm{CH}_{2}\right), 2.48(\mathrm{t}, 2 \mathrm{H}, J 7.7 \mathrm{~Hz}$, $\left.\mathrm{CH}_{2}\right), 2.86-3.06\left(\mathrm{~m}, 2 \mathrm{H}, \mathrm{CH}_{2}\right), 3.22-3.32\left(\mathrm{~m}, 3 \mathrm{H}, \mathrm{NH}_{2}\right.$ and $\mathrm{OH}), 3.89-3.98\left(\mathrm{~m}, 3 \mathrm{H}, \mathrm{CH}_{2}\right.$ and $\left.\mathrm{CH}\right), 6.57-6.64(\mathrm{~m}, 4 \mathrm{H}$, Ar-H), 6.67 (d, 1H, J 7.6 Hz, Ar-H), 7.09 (t, $1 \mathrm{H}, J 7.7 \mathrm{~Hz}$, Ar-H), 7.18-7.22 (m, 2H, Ar-H); ${ }^{13} \mathrm{C}$ NMR $(75 \mathrm{MHz}$, $\left.\mathrm{CDCl}_{3}\right) \delta$ 14.1, 22.7, 29.4, 29.5, 29.6, 29.7, 31.4, 31.9, 36.0, 40.0, 68.4, 70.2, 111.5, 114.8, 116.3, 121.3, 129.1, 134.0, 144.7, 158.5; HRMS (ESI) $\mathrm{m} / z$, calcd. for $\mathrm{C}_{30} \mathrm{H}_{48} \mathrm{NO}_{2} \mathrm{~S}^{+}$ $[\mathrm{M}+\mathrm{H}]^{+}$: 486.3400, found: 486.3404 .

1-(Ethylthio)-3-(3-pentadecylphenoxy)propan-2-ol (5j)

Yield: 95\%; white solid; ${ }^{1} \mathrm{H}$ NMR $\left(300 \mathrm{MHz}, \mathrm{CDCl}_{3}\right.$ ) $\delta 0.80\left(\mathrm{t}, 3 \mathrm{H}, J 6.3 \mathrm{~Hz}, \mathrm{CH}_{3}\right), 1.18-1.23\left(\mathrm{~m}, 27 \mathrm{H}, 12 \mathrm{CH}_{2}\right.$ and $\left.\mathrm{CH}_{3}\right), 1.49-1.54\left(\mathrm{~m}, 2 \mathrm{H}, \mathrm{CH}_{2}\right), 2.46-2.51(\mathrm{~m}, 5 \mathrm{H})$, 2.67-2.82 (m, 2H, $\left.\mathrm{CH}_{2}\right), 3.93-4.04\left(\mathrm{~m}, 3 \mathrm{H}, \mathrm{CH}_{2}\right.$ and $\left.\mathrm{CH}\right)$, 6.63-6.72 (m, 3H, Ar-H), 7.10 (t, 1H, J 7.7 Hz, Ar-H); ${ }^{13} \mathrm{C}$ NMR (75 MHz, $\left.\mathrm{CDCl}_{3}\right) \delta 14.1,14.8,22.7,26.4,29.3$, 29.5, 29.6, 29.7, 31.4, 31.9, 35.4, 35.9, 68.7, 70.3, 111.5, 114.8, 121.2, 129.2, 144.7, 158.4; HRMS (ESI) $\mathrm{m} / z$, calcd . for $\mathrm{C}_{26} \mathrm{H}_{47} \mathrm{O}_{2} \mathrm{~S}^{+}[\mathrm{M}+\mathrm{H}]^{+}:$: 423.3291, found 423.3297.

\section{1-Ethoxy-3-(3-pentadecylphenoxy)propan-2-ol (5k)}

Yield: $40 \%$; white solid; ${ }^{1} \mathrm{H}$ NMR $\left(300 \mathrm{MHz}, \mathrm{CDCl}_{3}\right)$ $\delta 0.80\left(\mathrm{t}, 3 \mathrm{H}, J 6.8 \mathrm{~Hz}, \mathrm{CH}_{3}\right), 1.12-1.26\left(\mathrm{~m}, 27 \mathrm{H}, 12 \mathrm{CH}_{2}\right.$ and $\left.\mathrm{CH}_{3}\right), 1.52-1.57\left(\mathrm{~m}, 2 \mathrm{H}, \mathrm{CH}_{2}\right), 2.34(\mathrm{br}, 1 \mathrm{H}, \mathrm{OH}), 2.49$ (t, $\left.2 \mathrm{H}, J 7.6 \mathrm{~Hz}, \mathrm{CH}_{2}\right), 3.45-3.58\left(\mathrm{~m}, 4 \mathrm{H}, \mathrm{CH}_{2}\right), 3.93-3.95$ (m, 2H, $\left.\mathrm{CH}_{2}\right)$, 4.05-4.12 (m, 1H, CH), 6.64-6.72 (m, 3H, Ar-H), 7.10 (t, $1 \mathrm{H}, J 7.6 \mathrm{~Hz}, \mathrm{Ar}-\mathrm{H}) ;{ }^{13} \mathrm{C}$ NMR $(75 \mathrm{MHz}$, $\left.\mathrm{CDCl}_{3}\right) \delta 14.1,15.1,22.7,29.3,29.5,29.6,29.7,31.4$, $31.9,36.0,66.9,68.9,69.1,71.3,111.4,114.8,121.3$, 129.2, 144.7, 158.6.

Synthesis of $\beta$-hydroxy sulfoxides $\mathbf{6 a - 6 \mathbf { j }}$ and sulfones $\mathbf{7 a}$, 7c-7f, $7 \mathrm{~h}-7 \mathrm{j}$

Aqueous $\mathrm{H}_{2} \mathrm{O}_{2}$ solution (4 $\mathrm{H}_{2} \mathrm{O}_{2}$ equivalents) was added to a reaction tube containing $1 \mathrm{mmol}$ of $\beta$-hydroxy sulfide (5a-5j) dissolved in acetic acid solution $(1 \mathrm{~mL})$ and the resulting reaction mixture was vigorously stirred at room temperature until completion of the reaction as judged by TLC. The solution was neutralized with $\mathrm{NaOH}$ and the product extracted with $\mathrm{CH}_{2} \mathrm{Cl}_{2}$. The organic phase was dried with anhydrous $\mathrm{Na}_{2} \mathrm{SO}_{4}$ and the material concentrated under reduced pressure. The crude mixture was fractionated in a chromatographic column with hexane:ethyl acetate (5:2) elution, resulting in compounds 6a-6j and 7a, 7c-7f, 7h-7j.

${ }^{1} \mathrm{H}$ NMR, ${ }^{13} \mathrm{C}$ NMR, and MS data of isolated compounds

1-(3-Pentadecylphenoxy)-3-(phenylsulfinyl)propan-2-ol (6a)

Yield: $60 \%$; white solid; ${ }^{1} \mathrm{H}$ NMR $\left(300 \mathrm{MHz}, \mathrm{CDCl}_{3}\right)$ $\delta 0.80\left(\mathrm{t}, 3 \mathrm{H}, J 6.8 \mathrm{~Hz}, \mathrm{CH}_{3}\right), 1.17-1.21\left(\mathrm{~m}, 24 \mathrm{H}, 12 \mathrm{CH}_{2}\right)$, 1.45-1.52 (m, 2H, $\left.\mathrm{CH}_{2}\right), 2.42-2.49\left(\mathrm{~m}, 2 \mathrm{H}, \mathrm{CH}_{2}\right), 2.88-3.13$ (m, 2H, $\left.\mathrm{CH}_{2}\right), 3.44$ (br, 1H, OH), 3.86-4.07 (m, 2H, $\mathrm{CH}_{2}$ ), 4.47-4.56 (m, $\left.2 \mathrm{H}, \mathrm{CH}_{2}\right), 6.55-6.71$ (m, 3H, Ar-H), 7.03-7.10 (m, 1H, Ar-H), 7.43-7.48 (m, 3H, Ar-H), 7.57-7.62 (m, 2H, $\mathrm{Ar}-\mathrm{H}) ;{ }^{13} \mathrm{C}$ NMR $\left(75 \mathrm{MHz}, \mathrm{CDCl}_{3}\right) \delta 14.1,22.6,29.3,29.4$, 29.5, 29.6, 31.3, 31.8, 35.9, 60.0, 65.1, 67.1, 70.3, 70.7, 111.4, 114.6, 114.7, 121.4, 121.5, 123.9, 124.0, 129.1, $129.2,129.3,129.4,131.1,131.4,142.9,143.4,144.6$, 144.7, 158.1, 158.2; HRMS (ESI) $\mathrm{m} / 2$, calcd. for $\mathrm{C}_{30} \mathrm{H}_{47} \mathrm{O}_{3} \mathrm{~S}^{+}$ $[\mathrm{M}+\mathrm{H}]^{+}$: 487.3240, found: 487.3224 .

1-(3-Pentadecylphenoxy)-3-(p-tolylsulfinyl)propan-2-ol (6b)

Yield: $75 \%$; white solid; ${ }^{1} \mathrm{H}$ NMR $\left(300 \mathrm{MHz}, \mathrm{CDCl}_{3}\right.$ ) $\delta 0.86\left(\mathrm{t}, 3 \mathrm{H}, J 6.9 \mathrm{~Hz}, \mathrm{CH}_{3}\right), 1.23-1.28\left(\mathrm{~m}, 24 \mathrm{H}, 12 \mathrm{CH}_{2}\right)$, 1.51-1.58 (m, $2 \mathrm{H}, \mathrm{CH}_{2}$ ), 2.41 (s, $3 \mathrm{H}, \mathrm{CH}_{3}$ ), 2.49-2.56 (m, $\left.2 \mathrm{H}, \mathrm{CH}_{2}\right), 2.85-2.91(\mathrm{~m}, 1 \mathrm{H}), 3.09\left(\mathrm{~m}, 2 \mathrm{H}, \mathrm{CH}_{2}\right), 3.90-$ $3.97\left(\mathrm{~m}, 1 \mathrm{H}, \mathrm{CH}_{2}\right), 4.08-4.13\left(\mathrm{~m}, 1 \mathrm{H}, \mathrm{CH}_{2}\right), 4.50-4.62$ (m, 1H, CH), 6.61-6.70 (m, 2H, Ar-H), 6.74-6.78 (m, 1H, Ar-H), 7.10-7.17 (m, 1H, Ar-H), 7.32-7.34 (m, 2H, Ar-H), 7.52-7.57 (m, 2H, Ar-H); ${ }^{13} \mathrm{C}$ NMR $\left(75 \mathrm{MHz}, \mathrm{CDCl}_{3}\right)$ $\delta$ 14.1, 21.4, 22.7, 29.4, 29.5, 29.6, 29.7, 31.4, 31.9, 35.9, 58.7, 59.7, 65.7, 67.5, 70.6, 111.4, 114.8, 121.5, 121.6, 124.0, 129.1, 129.2, 130.1, 130.2, 139.4, 141.8, 144.8, 158.2; HRMS (ESI) $m / z$, calcd. for $\mathrm{C}_{31} \mathrm{H}_{49} \mathrm{O}_{3} \mathrm{~S}^{+}[\mathrm{M}+\mathrm{H}]^{+}$: 501.3397, found: 501.3389 .

1-((4-Fluorophenyl)sulfinyl)-3-(3-pentadecylphenoxy) propan-2-ol (6c)

Yield: 55\%; white solid; ${ }^{1} \mathrm{H}$ NMR (300 $\mathrm{MHz}, \mathrm{CDCl}_{3}$ ) $\delta 0.80\left(\mathrm{t}, 3 \mathrm{H}, J 6.4 \mathrm{~Hz}, \mathrm{CH}_{3}\right), 1.18-1.25\left(\mathrm{~m}, 24 \mathrm{H}, 12 \mathrm{CH}_{2}\right)$, 1.45-1.48 (m, $\left.2 \mathrm{H}, \mathrm{CH}_{2}\right), 2.44-2.50\left(\mathrm{~m}, 2 \mathrm{H}, \mathrm{CH}_{2}\right), 2.84-3.14$ (m, $\left.2 \mathrm{H}, \mathrm{CH}_{2}\right), 3.38$ (br, $\left.1 \mathrm{H}, \mathrm{OH}\right), 3.87-4.08\left(\mathrm{~m}, 2 \mathrm{H}, \mathrm{CH}_{2}\right)$, 4.48-4.52 (m, 1H, CH), 6.56-6.64 (m, 2H, Ar-H), 6.69-6.73 (m, 1H, Ar-H), 7.07 (t, 1H, J 6.9 Hz, Ar-H), 7.14-7.17 (m, $2 \mathrm{H}, \mathrm{Ar}-\mathrm{H}), 7.57-7.65$ (m, 2H, Ar-H); ${ }^{13} \mathrm{C}$ NMR $(75 \mathrm{MHz}$, $\left.\mathrm{CDCl}_{3}\right) \delta 14.1,22.7,29.3,29.5,29.6,29.7,31.4,31.9$, $35.9,59.9,60.1,65.3,67.1,70.3,70.6,111.4,114.6$, $114.7,116.6,116.7,116.9,117.0,121.5,121.6,126.2$, 126.3, 126.4, 129.1, 129.2, 144.7, 144.8, 158.1, 162.9, 166.0; HRMS (ESI) $m / z$, calcd. for $\mathrm{C}_{30} \mathrm{H}_{46} \mathrm{FO}_{3} \mathrm{~S}^{+}[\mathrm{M}+\mathrm{H}]^{+}$: 505.3146, found: 505.3186 . 
1-((2-Chlorophenyl)sulfinyl)-3-(3-pentadecylphenoxy) propan-2-ol (6d)

Yield: 67\%; white solid; ${ }^{1} \mathrm{H}$ NMR $\left(300 \mathrm{MHz}, \mathrm{CDCl}_{3}\right.$ ) $\delta 0.80\left(\mathrm{t}, 3 \mathrm{H}, J 6.9 \mathrm{~Hz}, \mathrm{CH}_{3}\right), 1.17-1.22\left(\mathrm{~m}, 23 \mathrm{H}, 11 \mathrm{CH}_{2}\right)$, $1.51\left(\mathrm{~m}, 2 \mathrm{H}, \mathrm{CH}_{2}\right), 2.43-2.50\left(\mathrm{~m}, 2 \mathrm{H}, \mathrm{CH}_{2}\right), 2.91-2.98(\mathrm{~m}$, $\left.1 \mathrm{H}, \mathrm{CH}_{2}\right), 3.38-3.51\left(\mathrm{~m}, 1 \mathrm{H}, \mathrm{CH}_{2}\right), 3.90-4.10\left(\mathrm{~m}, 2 \mathrm{H}, \mathrm{CH}_{2}\right)$, 4.47-4.68 (m, 1H, CH), 6.55-6.72 (m, 3H, Ar-H), 7.02-7.12 (m, 1H, Ar-H), 7.31-7.49 (m, 3H, Ar-H), 7.86-7.92 (m, 1H, $\mathrm{Ar}-\mathrm{H}) ;{ }^{13} \mathrm{C}$ NMR $\left(75 \mathrm{MHz}, \mathrm{CDCl}_{3}\right) \delta 14.1,22.6,28.5,29.3$, $29.4,29.5,29.6,31.3,31.7,31.9,35.9,55.7,56.8,65.6$, $67.1,70.4,70.5,111.3,111.4,114.7,121.4,121.5,125.7$ $126.3,127.9,128.1,129.1,129.2,129.8,129.9,130.0$, 132.2, 140.6, 141.6, 144.6, 144.7, 158.1, 158.2; HRMS (ESI) $\mathrm{m} / z$, calcd. for $\mathrm{C}_{30} \mathrm{H}_{46} \mathrm{ClO}_{3} \mathrm{~S}^{+}[\mathrm{M}+\mathrm{H}]^{+}:$521.2851, found: 521.2871 .

1-((3-Chlorophenyl)sulfinyl)-3-(3-pentadecylphenoxy) propan-2-ol (6e)

Yield: 54\%; white solid; ${ }^{1} \mathrm{H}$ NMR $\left(300 \mathrm{MHz}, \mathrm{CDCl}_{3}\right.$ ) $\delta 0.80$ (t, $\left.3 \mathrm{H}, J 6.5 \mathrm{~Hz}, \mathrm{CH}_{3}\right), 1.18-1.22\left(\mathrm{~m}, 24 \mathrm{H}, 12 \mathrm{CH}_{2}\right)$, 1.48-1.52 (m, $\left.2 \mathrm{H}, \mathrm{CH}_{2}\right), 2.44-2.51\left(\mathrm{~m}, 2 \mathrm{H}, \mathrm{CH}_{2}\right), 2.86-$ 2.91 (br, $1 \mathrm{H}, \mathrm{OH}), 3.06-3.17\left(\mathrm{~m}, 2 \mathrm{H}, \mathrm{CH}_{2}\right), 3.87-4.09$ $\left(\mathrm{m}, 2 \mathrm{H}, \mathrm{CH}_{2}\right), 4.47-4.53(\mathrm{~m}, 1 \mathrm{H}, \mathrm{CH}), 6.57-6.65(\mathrm{~m}$, $2 \mathrm{H}, \mathrm{Ar}-\mathrm{H}), 6.69-6.74(\mathrm{~m}, 1 \mathrm{H}, \mathrm{Ar}-\mathrm{H}), 7.05-7.13(\mathrm{~m}, 2 \mathrm{H}$, Ar-H), 7.39-7.49 (m, 3H, Ar-H), 7.61-7.63 (m, 1H, Ar-H); ${ }^{13} \mathrm{C} \mathrm{NMR}\left(75 \mathrm{MHz}, \mathrm{CDCl}_{3}\right) \delta 14.1,22.7,29.3,29.5,29.6$, 29.7, 31.4, 31.9, 35.9, 59.8, 59.9, 65.4, 67.1, 70.3, 70.5, 111.4, 114.6, 114.7, 121.6, 121.6, 122.0, 124.0, 124.1, $129.2,129.3,130.6,130.7,131.3,131.6,135.8,144.8$, 144.8, 145.0, 145.3, 158.1; HRMS (ESI) $\mathrm{m} / z$, calcd. for $\mathrm{C}_{30} \mathrm{H}_{46} \mathrm{ClO}_{3} \mathrm{~S}^{+}[\mathrm{M}+\mathrm{H}]^{+}:$521.2851, found: 521.2873.

1-((4-Chlorophenyl)sulfinyl)-3-(3-pentadecylphenoxy) propan-2-ol (6f)

Yield: 57\%; white solid; ${ }^{1} \mathrm{H}$ NMR (300 MHz, $\mathrm{CDCl}_{3}$ ) $\delta 0.80$ (t, $\left.3 \mathrm{H}, J 6.6 \mathrm{~Hz}, \mathrm{CH}_{3}\right), 1.18-1.22\left(\mathrm{~m}, 24 \mathrm{H}, 12 \mathrm{CH}_{2}\right)$, 1.46-1.53 (m, $\left.2 \mathrm{H}, \mathrm{CH}_{2}\right), 2.44-2.50\left(\mathrm{~m}, 2 \mathrm{H}, \mathrm{CH}_{2}\right), 2.83-$ $3.15\left(\mathrm{~m}, 2 \mathrm{H}, \mathrm{CH}_{2}\right), 3.24-3.39$ (br, $\left.1 \mathrm{H}, \mathrm{OH}\right), 3.86-4.07$ $\left(\mathrm{m}, 2 \mathrm{H}, \mathrm{CH}_{2}\right), 4.44-4.52(\mathrm{~m}, 1 \mathrm{H}, \mathrm{CH}), 6.55-6.64(\mathrm{~m}, 2 \mathrm{H}$, Ar-H), 6.69-6.73 (m, 1H, Ar-H), 7.05-7.12 (m, 1H, Ar$\mathrm{H})$, 7.43-7.46 (m, 2H, Ar-H), 7.51-7.57 (m, 2H, Ar-H); ${ }^{13} \mathrm{C} \mathrm{NMR}\left(75 \mathrm{MHz}, \mathrm{CDCl}_{3}\right) \delta 14.1,22.7,29.3,29.5,29.6$, 29.7, 31.4, 31.9, 35.9, 59.7, 59.9, 65.3, 67.1, 70.3, 70.5, $111.4,114.7,114.7,121.6,121.6,125.4,125.4,129.2$, 129.3, 129.7, 129.8, 137.5, 137.7, 141.5, 142.0, 144.7, 144.8, 158.1; HRMS (ESI) $\mathrm{m} / z$, calcd. for $\mathrm{C}_{30} \mathrm{H}_{46} \mathrm{ClO}_{3} \mathrm{~S}^{+}$ $[\mathrm{M}+\mathrm{H}]^{+}:$521.2851, found: 521.2873 .

1-((2-Bromophenyl)sulfinyl)-3-(3-pentadecylphenoxy) propan-2-ol (6g)

Yield: $60 \%$; white solid; ${ }^{1} \mathrm{H}$ NMR $\left(300 \mathrm{MHz}, \mathrm{CDCl}_{3}\right.$ ) $\delta 0.81\left(\mathrm{t}, 3 \mathrm{H}, J 6.4 \mathrm{~Hz}, \mathrm{CH}_{3}\right), 1.18-1.23\left(\mathrm{~m}, 24 \mathrm{H}, 12 \mathrm{CH}_{2}\right)$, 1.48-1.54 (m, 2H, $\left.\mathrm{CH}_{2}\right), 2.49$ (t, $2 \mathrm{H}, J 7.4 \mathrm{~Hz}, \mathrm{CH}_{2}$ ), 2.88-2.98 (m, $\left.1 \mathrm{H}, \mathrm{CH}_{2}\right), 3.43-3.57\left(\mathrm{~m}, 1 \mathrm{H}, \mathrm{CH}_{2}\right), 3.91-4.11$ (m, 2H, $\left.\mathrm{CH}_{2}\right), 4.45-4.68(\mathrm{~m}, 1 \mathrm{H}, \mathrm{CH}), 6.56-6.73(\mathrm{~m}, 3 \mathrm{H}$, Ar-H), 7.13 (t, 1H, J 7.9 Hz, Ar-H), 7.30-7.36 (m, 1H, Ar-H), 7.49-7.56 (m, 2H, Ar-H), 7.87-7.92 (m, 1H, Ar-H); ${ }^{13} \mathrm{C} \mathrm{NMR}\left(75 \mathrm{MHz}, \mathrm{CDCl}_{3}\right) \delta 14.1,22.7,29.3,29.5,29.6$, 29.7, 31.4, 31.9, 35.9, 56.9, 65.9, 67.3, 70.4, 70.5, 111.5, $114.8,118.4,121.6,126.2,126.8,128.6,128.8,129.2$, $132.5,133.0,133.2,143.5,144.8,158.1$; HRMS (ESI) $\mathrm{m} / z$, calcd. for $\mathrm{C}_{30} \mathrm{H}_{46} \mathrm{BrO}_{3} \mathrm{~S}^{+}[\mathrm{M}+\mathrm{H}]^{+}: 565.2346$, found: 565.2348 .

1-((4-Bromophenyl)sulfinyl)-3-(3-pentadecylphenoxy) propan-2-ol (6h)

Yield: 59\%; white solid; ${ }^{1} \mathrm{H}$ NMR (300 $\mathrm{MHz}, \mathrm{CDCl}_{3}$ ) $\delta 0.81\left(\mathrm{t}, 3 \mathrm{H}, J 6.5 \mathrm{~Hz}, \mathrm{CH}_{3}\right), 1.18-1.22\left(\mathrm{~m}, 24 \mathrm{H}, 12 \mathrm{CH}_{2}\right)$, 1.47-1.53 (m, $\left.2 \mathrm{H}, \mathrm{CH}_{2}\right), 2.44-2.51\left(\mathrm{~m}, 2 \mathrm{H}, \mathrm{CH}_{2}\right), 2.83-3.15$ (m, 3H, $\left.\mathrm{CH}_{2}\right), 3.86-4.08\left(\mathrm{~m}, 2 \mathrm{H}, \mathrm{CH}_{2}\right), 4.45-4.53(\mathrm{~m}, 1 \mathrm{H}$, $\mathrm{CH}), 6.56-6.64$ (m, 2H, Ar-H), 6.72 (d, 1H, J 7.2 Hz, Ar-H), 7.08 (t, 2H, J 7.1 Hz, Ar-H), 7.46 (t, 2H, J 8.6 Hz, Ar-H), 7.59-7.62 (m, 2H, Ar-H); ${ }^{13} \mathrm{C}$ NMR $\left(75 \mathrm{MHz}, \mathrm{CDCl}_{3}\right.$ ) $\delta$ 14.1, 22.7, 29.3, 29.5, 29.6, 29.7, 31.4, 31.9, 35.9, 59.5, $59.8,65.4,67.1,70.3,70.5,111.4,114.7,114.7,121.6$, 121.6, 125.6, 125.7, 125.9, 129.2, 129.3, 132.6, 132.7, 144.8, 158.1; HRMS (ESI) $\mathrm{m} / z$, calcd. for $\mathrm{C}_{30} \mathrm{H}_{46} \mathrm{BrO}_{3} \mathrm{~S}^{+}$ $[\mathrm{M}+\mathrm{H}]^{+}:$565.2346, found: 565.2368 .

1-((4-Aminophenyl)sulfinyl)-3-(3-pentadecylphenoxy) propan-2-ol (6i)

Yield: 45\%; light yellow oil; ${ }^{1} \mathrm{H}$ NMR $(300 \mathrm{MHz}$, $\left.\mathrm{CDCl}_{3}\right) \delta 0.86\left(\mathrm{t}, 3 \mathrm{H}, J 6.2 \mathrm{~Hz}, \mathrm{CH}_{3}\right), 1.23(\mathrm{~m}, 24 \mathrm{H}$, $\left.12 \mathrm{CH}_{2}\right), 1.52-1.57\left(\mathrm{~m}, 2 \mathrm{H}, \mathrm{CH}_{2}\right), 2.52(\mathrm{t}, 2 \mathrm{H}, J 6.7 \mathrm{~Hz}$, $\left.\mathrm{CH}_{2}\right), 2.88-3.18\left(\mathrm{~m}, 2 \mathrm{H}, \mathrm{CH}_{2}\right), 3.88-4.11(\mathrm{~m}, 3 \mathrm{H}, \mathrm{OH}$ and $\left.\mathrm{CH}_{2}\right), 4.48-4.58(\mathrm{~m}, 1 \mathrm{H}, \mathrm{CH}), 6.62-6.77(\mathrm{~m}, 5 \mathrm{H}$, Ar-H), 7.10-7.16 (m, 1H, Ar-H), 7.41-7.47 (m, 2H, Ar-H); ${ }^{13} \mathrm{C} \mathrm{NMR}\left(75 \mathrm{MHz}, \mathrm{CDCl}_{3}\right) \delta 14.1,22.7,29.3,29.5,29.6$, 29.7, 31.4, 31.9, 35.9, 58.9, 59.6, 65.6, 67.4, 70.4, 70.7, $111.4,114.7,114.8,115.1,115.2,121.4,126.1,126.4$, 129.2, 144.7, 149.7, 150.0, 158.2; HRMS (ESI) $\mathrm{m} / z$, calcd. for $\mathrm{C}_{30} \mathrm{H}_{48} \mathrm{NO}_{3} \mathrm{~S}^{+}[\mathrm{M}+\mathrm{H}]^{+}: 502.3349$, found: 502.3319 .

1-(Ethylsulfinyl)-3-(3-pentadecylphenoxy)propan-2-ol (6j)

Yield: $65 \%$; white solid; ${ }^{1} \mathrm{H}$ NMR $\left(300 \mathrm{MHz}, \mathrm{CDCl}_{3}\right.$ ) $\delta 0.81\left(\mathrm{t}, 3 \mathrm{H}, J 6.6 \mathrm{~Hz}, \mathrm{CH}_{3}\right), 1.14-1.33\left(\mathrm{~m}, 3 \mathrm{H}, \mathrm{CH}_{2}\right.$ and $\left.\mathrm{CH}_{3}\right), 1.50-1.55\left(\mathrm{~m}, 2 \mathrm{H}, \mathrm{CH}_{2}\right), 1.69(\mathrm{br}, 1 \mathrm{H}, \mathrm{OH}), 2.49$ (t, $\left.2 \mathrm{H}, J 7.7 \mathrm{~Hz}, \mathrm{CH}_{2}\right), 2.72-3.01\left(\mathrm{~m}, 4 \mathrm{H}, \mathrm{CH}_{2}\right), 3.86-4.10$ $\left(\mathrm{m}, 3 \mathrm{H}, \mathrm{CH}_{2}\right), 4.54-4.62(\mathrm{~m}, 1 \mathrm{H}, \mathrm{CH}), 6.63-6.67(\mathrm{~m}, 2 \mathrm{H}$, Ar-H), 6.73 (d, 1H, J 7.6 Hz, Ar-H), $7.12(\mathrm{t}, 1 \mathrm{H}, J 7.7 \mathrm{~Hz})$; ${ }^{13} \mathrm{C}$ NMR $\left(75 \mathrm{MHz}, \mathrm{CDCl}_{3}\right) \delta 6.9,14.1,22.7,29.3,29.5$, 29.6, 29.7, 31.4, 31.9, 35.9, 46.0, 52.9, 54.0, 65.3, 67.0, 
111.4, 114.7, 121.6, 129.3, 144.8, 158.2; HRMS (ESI) $m / z$, calcd. for $\mathrm{C}_{26} \mathrm{H}_{47} \mathrm{O}_{3} \mathrm{~S}^{+}[\mathrm{M}+\mathrm{H}]^{+}:$: 439.3240, found: 439.3262 .

1-(3-Pentadecylphenoxy)-3-(phenylsulfonyl)propan-2-ol (7a)

Yield: 30\%; white solid; ${ }^{1} \mathrm{H}$ NMR (300 $\mathrm{MHz}, \mathrm{CDCl}_{3}$ ) $\delta 0.80\left(\mathrm{t}, 3 \mathrm{H}, J 6.6 \mathrm{~Hz}, \mathrm{CH}_{3}\right), 1.18-1.22\left(\mathrm{~m}, 24 \mathrm{H}, 12 \mathrm{CH}_{2}\right)$, 1.47-1.52 (m, 2H, $\left.\mathrm{CH}_{2}\right), 2.47\left(\mathrm{t}, 2 \mathrm{H}, J 7.7 \mathrm{~Hz}, \mathrm{CH}_{2}\right)$, 3.33-3.45 (m, 2H, $\left.\mathrm{CH}_{2}\right), 3.88-3.97\left(\mathrm{~m}, 2 \mathrm{H}, \mathrm{CH}_{2}\right), 4.41-4.49$ $(\mathrm{m}, 1 \mathrm{H}, \mathrm{CH}), 6.55-6.60(\mathrm{~m}, 2 \mathrm{H}, \mathrm{Ar}-\mathrm{H}), 6.71(\mathrm{~d}, 1 \mathrm{H}$, $J 7.5 \mathrm{~Hz}, \mathrm{Ar}-\mathrm{H}), 7.08$ (t, 1H, J 7.5 Hz, Ar-H), 7.49-7.54 (m, 2H, Ar-H), 7.58-7.63 (m, 1H, Ar-H), 7.88-7.91 (m, 2H, $\mathrm{Ar}-\mathrm{H}) ;{ }^{13} \mathrm{C} \mathrm{NMR}\left(75 \mathrm{MHz}, \mathrm{CDCl}_{3}\right) \delta 14.1,22.7,29.3,29.3$, 29.5, 29.5, 29.6, 31.3, 31.9, 35.9, 59.4, 65.1, 69.9, 111.4, $114.7,121.7,128.0,129.2,129.4,134.1,139.1,144.8$, 157.9; HRMS (ESI) $m / z$, calcd. for $\mathrm{C}_{30} \mathrm{H}_{47} \mathrm{O}_{4} \mathrm{~S}^{+}[\mathrm{M}+\mathrm{H}]^{+}$: 503.3190, found: 503.3188.

1-((4-Fluorophenyl)sulfonyl)-3-(3-pentadecylphenoxy) propan-2-ol (7c)

Yield: 45\%; white solid; ${ }^{1} \mathrm{H}$ NMR (300 $\left.\mathrm{MHz}, \mathrm{CDCl}_{3}\right)$ $\delta 0.81\left(\mathrm{t}, 3 \mathrm{H}, J 6.6 \mathrm{~Hz}, \mathrm{CH}_{3}\right), 1.18-1.23\left(\mathrm{~m}, 24 \mathrm{H}, 12 \mathrm{CH}_{2}\right)$, 1.48-1.53 (m, 2H, $\left.\mathrm{CH}_{2}\right), 2.47\left(\mathrm{t}, 2 \mathrm{H}, J 7.7 \mathrm{~Hz}, \mathrm{CH}_{2}\right)$, 3.32-3.43 (m, 2H, $\left.\mathrm{CH}_{2}\right), 3.89-3.98\left(\mathrm{~m}, 2 \mathrm{H}, \mathrm{CH}_{2}\right), 4.42-4.49$ $(\mathrm{m}, 1 \mathrm{H}, \mathrm{CH}), 6.56-6.60(\mathrm{~m}, 2 \mathrm{H}, \mathrm{Ar}-\mathrm{H}), 6.72(\mathrm{~d}, 1 \mathrm{H}$, J 7.5 Hz, Ar-H), 7.09 (t, 1H, J 7.5 Hz, Ar-H), 7.16-7.22 (m, 2H, Ar-H), 7.89-7.95 (m, 2H, Ar-H); ${ }^{13} \mathrm{C}$ NMR (75 MHz, $\left.\mathrm{CDCl}_{3}\right) \delta 14.1,22.7,29.3,29.5,29.6,29.7,31.4,31.9,35.9$, 59.6, 65.2, 69.8, 111.4, 114.7, 116.6, 116.9, 121.8, 129.3, 130.9, 131.0, 144.9, 157.9; HRMS (ESI) $\mathrm{m} / z$, calcd. for $\mathrm{C}_{30} \mathrm{H}_{46} \mathrm{FO}_{4} \mathrm{~S}^{+}[\mathrm{M}+\mathrm{H}]^{+}:$521.3095, found: 521.3087 .

1-((2-Chlorophenyl)sulfonyl)-3-(3-pentadecylphenoxy) propan-2-ol (7d)

Yield: 23\%; white solid; ${ }^{1} \mathrm{H}$ NMR (300 $\mathrm{MHz}, \mathrm{CDCl}_{3}$ ) $\delta 0.81\left(\mathrm{t}, 3 \mathrm{H}, J 6.8 \mathrm{~Hz}, \mathrm{CH}_{3}\right), 1.18\left(\mathrm{~m}, 20 \mathrm{H}, 10 \mathrm{CH}_{2}\right), 1.51$ $\left(\mathrm{m}, 6 \mathrm{H}, 3 \mathrm{CH}_{2}\right), 2.48\left(\mathrm{t}, 2 \mathrm{H}, J 7.7 \mathrm{~Hz}, \mathrm{CH}_{2}\right), 3.63-3.82$ (m, 2H, $\left.\mathrm{CH}_{2}\right), 3.89-3.99\left(\mathrm{~m}, 2 \mathrm{H}, \mathrm{CH}_{2}\right), 4.43-4.45(\mathrm{~m}, 1 \mathrm{H}$, $\mathrm{CH}), 6.56-6.60$ (m, 2H, Ar-H), 6.71 (d, 1H, J 7.6 Hz, ArH), 7.09 (t, 1H, J 7.6 Hz, Ar-H), 7.39-7.44 (m, 1H, Ar-H), 7.47-7.52 (m, 2H, Ar-H), 8.08-8.11 (m, 1H, Ar-H); HRMS (ESI) $m / z$, calcd. for $\mathrm{C}_{30} \mathrm{H}_{46} \mathrm{ClO}_{4} \mathrm{~S}^{+}[\mathrm{M}+\mathrm{H}]^{+}$: 537.2800, found: 537.2798 .

1-((3-Chlorophenyl)sulfonyl)-3-(3-pentadecylphenoxy) propan-2-ol (7e)

Yield: 36\%; white solid; ${ }^{1} \mathrm{H}$ NMR (300 $\mathrm{MHz}, \mathrm{CDCl}_{3}$ ) $\delta 0.81\left(\mathrm{t}, 3 \mathrm{H}, J 6.5 \mathrm{~Hz}, \mathrm{CH}_{3}\right), 1.18-1.23\left(\mathrm{~m}, 24 \mathrm{H}, 12 \mathrm{CH}_{2}\right)$, 1.48-1.53 (m, 2H, $\left.\mathrm{CH}_{2}\right), 2.48\left(\mathrm{t}, 2 \mathrm{H}, J 7.7 \mathrm{~Hz}, \mathrm{CH}_{2}\right)$, 3.35-3.46 (m, 2H, $\left.\mathrm{CH}_{2}\right), 3.90-3.99\left(\mathrm{~m}, 2 \mathrm{H}, \mathrm{CH}_{2}\right), 4.44-4.51$ $(\mathrm{m}, 1 \mathrm{H}, \mathrm{CH}), 6.57-6.61(\mathrm{~m}, 2 \mathrm{H}, \mathrm{Ar}-\mathrm{H}), 6.72(\mathrm{~d}, 1 \mathrm{H}$,
$J 7.7 \mathrm{~Hz}, \mathrm{Ar}-\mathrm{H}), 7.09$ (t, 1H, J 7.7 Hz, Ar-H), 7.46 (t, 1H, J 7.9 Hz, Ar-H), 7.57 (d, 1H, J $8.8 \mathrm{~Hz}, \mathrm{Ar}-\mathrm{H}), 7.78$ (d, 1H, J $7.9 \mathrm{~Hz}, \mathrm{Ar}-\mathrm{H}), 7.90$ (s, 1H, Ar-H); ${ }^{13} \mathrm{C} \mathrm{NMR}(75 \mathrm{MHz}$, $\left.\mathrm{CDCl}_{3}\right) \delta 14.1,22.7,29.3,29.6,29.7,31.4,31.9,59.5$, 65.2, 69.8, 111.4, 114.8, 116.6, 121.8, 126.1, 128.2, 129.3, 130.7, 134.2, 142.8, 156.4; HRMS (ESI) $\mathrm{m} / z$, calcd. for $\mathrm{C}_{30} \mathrm{H}_{46} \mathrm{ClO}_{4} \mathrm{~S}^{+}[\mathrm{M}+\mathrm{H}]^{+}:$537.2800, found: 537.2808.

1-((4-Chlorophenyl)sulfonyl)-3-(3-pentadecylphenoxy) propan-2-ol (7f)

Yield: 38\%; white solid; ${ }^{1} \mathrm{H}$ NMR $\left(300 \mathrm{MHz}, \mathrm{CDCl}_{3}\right)$ $\delta 0.81\left(\mathrm{t}, 3 \mathrm{H}, J 6.6 \mathrm{~Hz}, \mathrm{CH}_{3}\right), 1.18-1.23\left(\mathrm{~m}, 22 \mathrm{H}, 11 \mathrm{CH}_{2}\right)$, 1.50-1.53 (m, 4H, 2CH $), 2.48\left(\mathrm{t}, 2 \mathrm{H}, J 7.7 \mathrm{~Hz}, \mathrm{CH}_{2}\right), 3.14$ (br, 1H, OH), 3.32-3.44 (m, 2H, $\left.\mathrm{CH}_{2}\right), 3.89-3.98$ (m, 2H, $\mathrm{CH}_{2}$ ), 4.41-4.48 (m, 1H, CH), 6.56-6.60 (m, 2H, Ar-H), 6.72 (d, 1H, J 7.6 Hz, Ar-H), 7.09 (t, 1H, J 7.6 Hz, Ar-H), 7.49 (d, 2H, J 8.7 Hz, Ar-H), 7.83 (d, 2H, J 8.7 Hz, Ar-H); ${ }^{13} \mathrm{C} \mathrm{NMR}\left(75 \mathrm{MHz}, \mathrm{CDCl}_{3}\right) \delta 14.1,22.7,29.3,29.5,29.6$, 29.7, 31.4, 31.9, 35.9, 59.6, 65.2, 69.8, 111.4, 114.7, 121.8, 129.3, 129.5, 129.8, 137.7, 140.9, 144.9, 158.1; HRMS (ESI) $\mathrm{m} / z$, calcd. for $\mathrm{C}_{30} \mathrm{H}_{46} \mathrm{ClO}_{4} \mathrm{~S}^{+}[\mathrm{M}+\mathrm{H}]^{+}$: 537.2800, found: 537.2808 .

1-((4-Bromophenyl)sulfonyl)-3-(3-pentadecylphenoxy) propan-2-ol (7h)

Yield: 33\%; white solid; ${ }^{1} \mathrm{H}$ NMR $\left(300 \mathrm{MHz}, \mathrm{CDCl}_{3}\right)$ $\delta 0.78\left(\mathrm{t}, 3 \mathrm{H}, J 6.5 \mathrm{~Hz}, \mathrm{CH}_{3}\right), 1.18-1.22\left(\mathrm{~m}, 24 \mathrm{H}, 12 \mathrm{CH}_{2}\right)$, 1.47-1.53 (m, 2H, $\left.\mathrm{CH}_{2}\right), 2.47\left(\mathrm{t}, 2 \mathrm{H}, J 7.7 \mathrm{~Hz}, \mathrm{CH}_{2}\right)$, 3.37-3.39 (m, 2H, $\left.\mathrm{CH}_{2}\right), 3.88-3.97\left(\mathrm{~m}, 2 \mathrm{H}, \mathrm{CH}_{2}\right), 4.41-4.48$ (m, 1H, CH), 6.55-6.60 (m, 2H, Ar-H), $6.71(\mathrm{~d}, 1 \mathrm{H}$, $J 7.5 \mathrm{~Hz}, \mathrm{Ar}-\mathrm{H}), 7.09$ (t, 1H, J 7.7 Hz, Ar-H), 7.65 (d, 2H, $J 8.6 \mathrm{~Hz}, \mathrm{Ar}-\mathrm{H}), 7.75$ (d, 2H, J 8.6 Hz, Ar-H); ${ }^{13} \mathrm{C} \mathrm{NMR}$ $\left(75 \mathrm{MHz}, \mathrm{CDCl}_{3}\right) \delta 14.1,22.7,29.3,29.5,29.6,29.7,31.4$, 31.9, 35.9, 59.5, 65.2, 69.8, 111.4, 114.7, 121.8, 129.3, 129.5, 129.6, 132.7, 138.2, 144.9, 157.8; HRMS (ESI) $m / z$, calcd. for $\mathrm{C}_{30} \mathrm{H}_{46} \mathrm{BrO}_{4} \mathrm{~S}^{+}[\mathrm{M}+\mathrm{H}]^{+}: 581.2295$, found: 581.2297 .

1-((4-Aminophenyl)sulfonyl)-3-(3-pentadecylphenoxy) propan-2-ol (7i)

Yield: 30\%; light yellow oil; ${ }^{1} \mathrm{H}$ NMR $(300 \mathrm{MHz}$, $\left.\mathrm{CDCl}_{3}\right) \delta 0.85\left(\mathrm{t}, 3 \mathrm{H}, J 6.8 \mathrm{~Hz}, \mathrm{CH}_{3}\right), 1.23-1.30(\mathrm{~m}, 24 \mathrm{H}$, $\left.12 \mathrm{CH}_{2}\right), 1.52-1.58\left(\mathrm{~m}, 2 \mathrm{H}, \mathrm{CH}_{2}\right), 2.52(\mathrm{t}, 2 \mathrm{H}, J 7.4 \mathrm{~Hz}$, $\left.\mathrm{CH}_{2}\right)$, 2.95-3.50 (m, 2H, $\left.\mathrm{CH}_{2}\right), 3.92-4.02\left(\mathrm{~m}, 2 \mathrm{H}, \mathrm{CH}_{2}\right)$, 4.42-4.56 (m, 1H, CH), 6.62-6.77 (m, 5H, Ar-H), 7.11-7.24 (m, 1H, Ar-H), 7.85 (d, 2H, J $8.6 \mathrm{~Hz}, \mathrm{Ar}-\mathrm{H}) ;{ }^{13} \mathrm{C} \mathrm{NMR}$ $\left(75 \mathrm{MHz}, \mathrm{CDCl}_{3}\right) \delta 14.1,22.7,29.4,29.5,29.6,29.7,31.4$, 31.9, 35.9, 59.7, 65.3, 70.0, 111.4, 114.2, 114.8, 121.6, 129.2, 130.2, 144.9, 151.8, 158.1; HRMS (ESI) $\mathrm{m} / \mathrm{z}$, calcd. for $\mathrm{C}_{30} \mathrm{H}_{48} \mathrm{NO}_{4} \mathrm{~S}^{+}[\mathrm{M}+\mathrm{H}]^{+}:$518.3299, found: 518.3287 . 
1-(Ethylsulfonyl)-3-(3-pentadecylphenoxy)propan-2-ol (7j)

Yield: $15 \%$; white solid; ${ }^{1} \mathrm{H}$ NMR $\left(300 \mathrm{MHz}, \mathrm{CDCl}_{3}\right)$ $\delta 0.86\left(\mathrm{t}, 3 \mathrm{H}, J 6.5 \mathrm{~Hz}, \mathrm{CH}_{3}\right), 1.23-1.28\left(\mathrm{~m}, 25 \mathrm{H}, 12 \mathrm{CH}_{2}\right)$, $1.42\left(\mathrm{t}, 3 \mathrm{H}, J 7.5 \mathrm{~Hz}, \mathrm{CH}_{3}\right), 1.55-1.60\left(\mathrm{~m}, 2 \mathrm{H}, \mathrm{CH}_{2}\right), 2.55$ (t, $\left.2 \mathrm{H}, J 7.7 \mathrm{~Hz}, \mathrm{CH}_{2}\right), 2.60-2.80(\mathrm{br}, 1 \mathrm{H}, \mathrm{OH}), 3.12-3.35$ (m, 4H, $\left.\mathrm{CH}_{2}\right), 3.97-4.05\left(\mathrm{~m}, 2 \mathrm{H}, \mathrm{CH}_{2}\right), 4.58-4.65(\mathrm{~m}, 1 \mathrm{H}$, $\mathrm{CH}), 6.67-6.71$ (m, 2H, Ar-H), 6.80 (d, 1H, J 7.7Hz, Ar-H), 7.17 (t, 1H, J 7.7 Hz, Ar-H); ${ }^{13} \mathrm{C}$ NMR (75 MHz, $\mathrm{CDCl}_{3}$ ) $\delta 6.5,14.1,22.7,29.3,29.5,29.6,29.7,31.4,31.9,35.9$, 49.0, 55.1, 65.4, 70.1, 111.4, 114.7, 121.8, 129.3, 144.9, 157.9; HRMS (ESI) $m / z$, calcd. for $\mathrm{C}_{26} \mathrm{H}_{47} \mathrm{O}_{4} \mathrm{~S}^{+}[\mathrm{M}+\mathrm{H}]^{+}$: 455.3190, found: 455.3182 .

\section{Antibacterial assays}

Antimicrobial potential was evaluated as per Micheletti et $a l .{ }^{26}$ Briefly, dimethyl sulfoxide (DMSO) solutions of samples were serially diluted in 96-well plates prepared with Mueller-Hinton broth (Sigma-Aldrich, São Paulo, Brazil) to reach final concentrations in the $1-1000 \mu \mathrm{g} \mathrm{mL} \mathrm{m}^{-1}$ range, with a final $100 \mu \mathrm{L}$ volume in each well. For gentamicin, the positive control, final concentrations ranged from 64 to $0.5 \mu \mathrm{g} \mathrm{mL} \mathrm{m}^{-1}$. The inocula consisted of overnight cultures of Staphylococcus aureus (NEWP0023) and Escherichia coli (NEWP0022) in Mueller-Hinton agar suspended in sterile saline solution $(0.45 \%)$ to a concentration of ca. $1 \times 10^{8}$ colony forming unit $(\mathrm{CFU}) \mathrm{mL}^{-1}$, then diluted $1: 10$ in saline solution $(0.45 \%)$, after which $5 \mu \mathrm{L}$ aliquots were added to each well containing the test samples. All experiments were performed in triplicate. The microdilution trays were incubated at $36{ }^{\circ} \mathrm{C}$ for $18 \mathrm{~h}$ and a $20 \mu \mathrm{L}$ volume of an aqueous solution $(0.5 \%)$ of triphenyl tetrazolium chloride (TTC) was added to each well, followed by tray incubation at $36{ }^{\circ} \mathrm{C}$ for another $2 \mathrm{~h}$. In those wells exhibiting bacterial growth, TTC changed from colorless to red. Minimum inhibitory concentration (MIC, expressed in micrograms per milliliter) was defined as the lowest concentration of each substance at which no color change occurred.

\section{In vitro antioxidant-activity assays}

Antioxidant activity was preliminarily evaluated via a spectrophotometric radical-scavenging method based on the 1,1-diphenyl-2-picrylhydrazyl (DPPH') radical, as adapted from Uriarte-Pueyo and Calvo. ${ }^{27} \mathrm{~A} 100 \mu \mathrm{L}$ volume of compound $\mathbf{5} \mathbf{a}-\mathbf{5} \mathbf{j}$ in the $500-1 \mu \mathrm{g} \mathrm{mL} \mathrm{L}^{-1}$ range was diluted in methanol and dispensed into a 96-well microplate in addition to $100 \mu \mathrm{L}$ of a $\mathrm{DPPH}^{\bullet}$ solution in methanol so as to obtain a final $\mathrm{DPPH}^{\bullet}$ concentration of $40 \mu \mathrm{g} \mathrm{mL}^{-1}$ in each well. After $30 \mathrm{~min}$ incubation protected from light at ambient temperature, absorbance readings at $492 \mathrm{~nm}$ obtained on an EZ Read 400 ELISA microplate reader (Biochrom) were used to calculate radical-scavenging activities (percent decrease of $\mathrm{DPPH}^{*}$, or $\mathrm{Q}$ ), applying the equation $\mathrm{Q}=100\left[\left(\mathrm{~A}_{\mathrm{c}}-\mathrm{A}_{0}\right) / \mathrm{A}_{0}\right]$, where $\mathrm{A}_{0}$ is the blank absorbance (DPPH ${ }^{\bullet}$ sample solution devoid of test compounds) and $\mathrm{A}_{\mathrm{c}}$ the absorbance for a $\mathrm{DPPH} \mathrm{H}^{\bullet}$ solution amended with the test compound at concentration $c$. Ascorbic acid, the positive control, was used at the same concentration range as the test compounds. The experiment was performed in triplicate.

\section{Results and Discussion}

\section{Synthesis}

The mixture of mono-, di-, and trienecardanols was obtained through reduced-pressure distillation of technicalgrade CNSL (tCNSL), which, subsequently subjected to catalytic hydrogenation, yielded saturated cardanol (1). This compound was then treated with epichlorohydrin (obtained from glycerol) to yield cardanol epoxide $\mathbf{3}$, as per Braga et al. ${ }^{11}$ The structure of $\mathbf{3}$ was confirmed by ${ }^{1} \mathrm{H}$ and ${ }^{13} \mathrm{C}$ NMR spectra and by comparison with published data. ${ }^{11,12}$

Initially, thiolysis was performed on cardanol epoxide $\mathbf{3}$ using an equimolar amount of thiophenol (4a) as a model substrate in water, ethanol, or both at ambient temperature and at 30 and $70{ }^{\circ} \mathrm{C}$ (Table 1$)$ in the presence and absence of a base. No reaction was observed when aqueous solution of a base was used at room temperature and $30^{\circ} \mathrm{C}$ (Table 1 , entries 1 and 2). After $24 \mathrm{~h}$ at $70{ }^{\circ} \mathrm{C}$, however, the yield was $15 \%$ (entry 3). At this temperature, however, Mukherjee et al..$^{23}$ thiolyzed epoxides in water using 2.5 thiophenol equivalents and obtained excellent yields in $5 \mathrm{~h}$ reaction. Our low yield can be partly explained by the use of only one thiol 4a equivalent, although the low solubility of cardanol epoxide $\mathbf{3}$ in water may also have played a role.

To generate a corresponding salt, thiophenol was treated with $2 \mathrm{~mL}$ of an aqueous $\mathrm{NaOH}$ solution $\left(0.03 \mathrm{~mol} \mathrm{~L}^{-1}\right)$ at $30{ }^{\circ} \mathrm{C}$ for $10 \mathrm{~min}$, with subsequent addition of epoxide $\mathbf{3}$, followed by stirring for another $2 \mathrm{~h}$, thus resulting in a $75 \%$ yield of the desired product (entry 4). Replacing water with ethanol increased the yield to $80 \%$ (entry 5).

The reaction was examined under optimized conditions (Table 1, entry 5). As seen in Table 2, yields ranged from good to excellent (61-95\%). All products were easily purified by silica gel column chromatography and chemical structures confirmed by ${ }^{1} \mathrm{H}$ NMR, ${ }^{13} \mathrm{C}$ NMR and HRMS.

Interestingly, formations of $\mathbf{5 b}$ (entry 2) and $\mathbf{5 i}$ (entry 9) was accompanied with generation of hydroxy ether $\mathbf{5 k}$, 
Table 1. Optimization of reaction conditions for thiolysis of cardanol epoxide $\mathbf{3}$ by thiophenol in water or ethanol

\begin{tabular}{|c|c|c|c|c|c|}
\hline & 3 & $4 a$ & & $5 a$ & \\
\hline entry & Solvent & Catalyst $^{\mathrm{a}}$ & Temperature $/{ }^{\circ} \mathrm{C}$ & time $/ \mathrm{h}$ & Yield $/ \%$ \\
\hline 1 & $\mathrm{H}_{2} \mathrm{O}$ & - & R.T. & 24 & $\mathrm{NR}^{\mathrm{c}}$ \\
\hline 2 & $\mathrm{H}_{2} \mathrm{O}$ & - & 30 & 24 & $\mathrm{NR}^{\mathrm{c}}$ \\
\hline 3 & $\mathrm{H}_{2} \mathrm{O}$ & - & 70 & 24 & 15 \\
\hline 4 & $\mathrm{H}_{2} \mathrm{O}$ & $\mathrm{NaOH}$ & 30 & 2 & 75 \\
\hline 5 & ethanol & $\mathrm{NaOH}$ & 30 & 2 & 80 \\
\hline
\end{tabular}

All reactions performed with $\mathbf{3}(1.0 \mathrm{mmol}), \mathbf{4 a}(1.0 \mathrm{mmol})$, catalyst, and solvent $(2 \mathrm{~mL})$ under heating conditions. ${ }^{2} 2 \mathrm{~mL}$ of $0.03 \mathrm{~mol} \mathrm{~L}{ }^{-1}$ ethanol or aqueous solution was employed; bisolated yield; 'no reaction. R.T.: room temperature.

Table 2. Thiolysis of cardanol epoxide 3 by using various thiols under ethanolic basic conditions

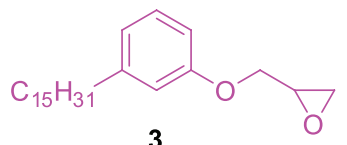

3

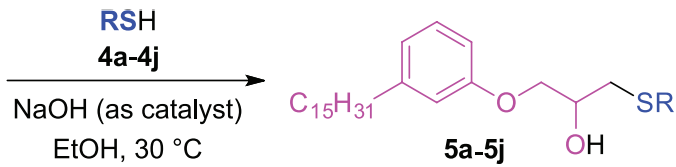

$\mathrm{EtOH}, 30^{\circ} \mathrm{C}$<smiles>CCCCCc1cccc(OCC(O)CSc2ccccc2)c1</smiles>

80

5a<smiles>CCCCCc1cccc(OCC(O)CSc2ccc(C)cc2)c1</smiles>

$5 \mathbf{b}$<smiles>[12CH2]c1cccc(OCC(O)CC(O)CSc2ccc(F)cc2)c1</smiles>

$5 \mathbf{c}$<smiles>CCCCCCCCCCCCCCCCCCCC(O)CSc1ccccc1Cl</smiles>

5d

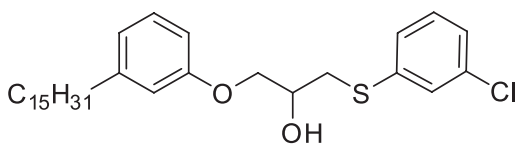

$5 e$<smiles>CCCCCCCCCCCCCCCCC(O)CSc1ccc(Cl)cc1</smiles> 
Table 2. Thiolysis of cardanol epoxide 3 by using various thiols under ethanolic basic conditions (cont.)

entry

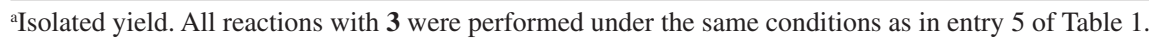

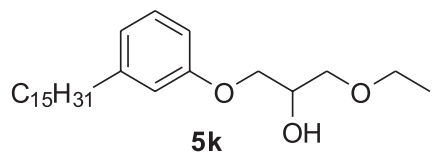

Figure 3. Chemical structure of compound $\mathbf{5 k}$.

owing to oxirane ring opening by ethanol. In both cases, 5k yield was around $40 \%$ (Figure 3 ).

The sulfoxides and corresponding sulfones for evaluation of antibacterial activity were obtained by oxidation of the previously synthesized $\beta$-hydroxy sulfides. In order to optimize the procedure, compound $\mathbf{5 a}$ was treated with 1 equivalent of peracetic acid, formed in situ by reacting $30 \% \mathrm{H}_{2} \mathrm{O}_{2}$ with glacial acetic acid at ambient temperature, so as to yield the corresponding sulfoxide $\mathbf{6 a}$, sulfone $\mathbf{7 a}$, or both. In $24 \mathrm{~h}$, only $60 \%$ substrate conversion was achieved, with $\mathbf{6 a}$ and $7 \mathbf{a}$ forming at a 9:1 ratio. Sulfone generation from sulfides generally involves two-step oxidation. Sulfide oxidation to sulfoxide is typically faster than the second step of sulfoxide-to-sulfone conversion, causing sulfoxide to be virtually the only product in most cases. However, as this was not the case in our experiments, we chose to investigate the synthesis of both compounds using an excess of the oxidizing agent, given that $6 \mathbf{a}$ and $7 \mathbf{a}$ would be easily separated by silica gel column chromatography. Treating $\mathbf{5 a}$ with 2 equivalents of the oxidizing agent produced $6 \mathbf{a}$ and 7a at an 8.5:1.5 ratio, with only $75 \%$ conversion of the substrate in $24 \mathrm{~h}$ at ambient temperature (entry 2). On the other hand, using 4 equivalents of $30 \% \mathrm{H}_{2} \mathrm{O}_{2}$ caused the substrate to be completely consumed in $12 \mathrm{~h}$, with a $\mathbf{6 a}: 7 \mathbf{a}$ ratio of 7:3 (Table 3 , entry 3 ).

The other $\beta$-hydroxy sulfoxides $(\mathbf{6 b}-\mathbf{6 j})$ and $\beta$-hydroxyl sulfones $(\mathbf{7} \mathbf{b}-\mathbf{7} \mathbf{j})$ were obtained in the same manner (Table 3, entry 3). Reaction time was in the range of 12-24 h, depending on substrate (Scheme 3). Easy to perform, the method proved useful for our purposes, facilitating separation of products.

Compounds $\mathbf{6 a - 6 j}$ and $\mathbf{7 a - 7} \mathbf{j}$ (except compounds $\mathbf{7 b}$ and $\mathbf{7 g}$ ) were purified in a silica gel column and had their structures confirmed by ${ }^{1} \mathrm{H}$ and ${ }^{13} \mathrm{C}$ NMR analysis. As expected, sulfoxides $\mathbf{6 a - 6 j}$ have two stereogenic centers,

Table 3. Optimization of reaction conditions for oxidation of $1 \mathrm{mmol}$ of compound $\mathbf{5 a}$ to sulfoxide $\mathbf{6 a}$ and sulfone $\mathbf{7 a}$, at ambient temperature using $30 \%$ $\mathrm{H}_{2} \mathrm{O}_{2}$ and $1 \mathrm{~mL}$ of glacial acetic acid

\begin{tabular}{lcccc}
\hline entry & $30 \% \mathrm{H}_{2} \mathrm{O}_{2} /$ equiv. & Substrate conversion $/ \%$ & time / h & 6a:7a ratio $^{\mathrm{b}}$ \\
\hline 1 & 1 & 60 & 24 & $9: 1$ \\
2 & 2 & 75 & 24 & $8.5: 1.5$ \\
3 & 4 & 100 & 12 & $7: 3$ \\
\hline
\end{tabular}

${ }^{\mathrm{a}}$ Calculated from recovered mass of starting material; ${ }^{\mathrm{b}}$ calculated from relative integrals of ${ }^{1} \mathrm{H}$ NMR spectra of mixture. 


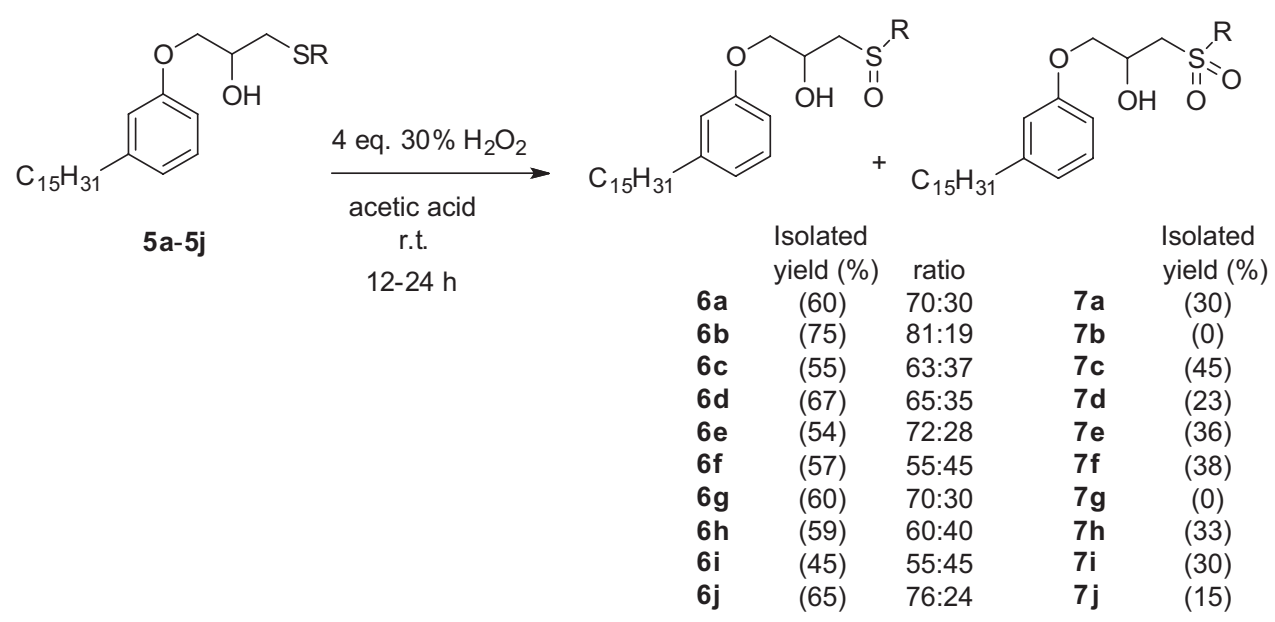

Scheme 3. Oxidation of sulfides $\mathbf{5 a}-\mathbf{5} \mathbf{j}$ to sulfoxides $\mathbf{6} \mathbf{a}-\mathbf{6 j}$ and sulfones $7 \mathbf{a}-\mathbf{7} \mathbf{j}$ by use of $\mathrm{H}_{2} \mathrm{O}_{2}$ as oxidant.

comprising the carbinol carbon with $R$ and $S$ configurations, as well as the sulfoxide group sulfur atom, making possible the formation of four stereoisomers. These are shown in Figure 4, with I and II exhibiting a diastereoisomeric relationship, while enantiomerism is observed between Ia and $\mathbf{I b}$, as well as between IIa and IIb.

Signals are superimposed in the ${ }^{1} \mathrm{H}$ NMR spectra of sulfoxides, whereas ${ }^{13} \mathrm{C}$ NMR signals are duplicated in all cases, confirming the presence of a mixture of diastereoisomers. Since classical chromatographic methods fail to separate stereoisomers, we chose to test sulfoxides 6a-6j for biological activity without prior separation of diastereoisomers.

\section{Antibacterial assays}

$\beta$-Hydroxy sulfides $\mathbf{5} \mathbf{a}-\mathbf{5} \mathbf{j}, \boldsymbol{\beta}$-hydroxy sulfoxides $\mathbf{6} \mathbf{a}-\mathbf{6 j}$, and $\beta$-hydroxy sulfones $\mathbf{7 a}, \mathbf{7 c - 7 f}, \mathbf{7 h}-\mathbf{7} \mathbf{j}$ were tested for antibacterial activity against standard strains of $S$. aureus (ATCC 25923, Gram-positive) and E. coli (ATCC 25922, Gram-negative) using previously reported methods ${ }^{12,26}$ (Table 4).

$\mathbf{5 a}, \mathbf{5 c}-\mathbf{5 e}$, and $\mathbf{5 h}-\mathbf{5} \mathbf{j}$ proved moderately active, ${ }^{29}$ with $\mathrm{MIC}=125 \mu \mathrm{g} \mathrm{mL} \mathrm{m}^{-1}$ against $S$. aureus, while $\mathbf{5 b}$ and $\mathbf{5 f}-\mathbf{5 g}$ and corresponding oxidized derivatives $\mathbf{6 a - 6 j}$ and $\mathbf{7 a}$, 7c-7f, $7 \mathbf{h}$-7j were inactive. $\beta$-Hydroxy sulfides had no effect on E. coli, a finding consistent with the fact that the outer membrane of Gram-negative bacteria can act as a barrier to lipophilic compounds. ${ }^{30,31}$ The membrane also protects enteric bacterial cells from the action of detergents (amphiphilic compounds such as the derivatives investigated here and in a previous study ${ }^{12}$ on $\beta$-amino lipophilic alcohols structurally similar to sulfur-containing derivatives). Compounds $\mathbf{5 a}, \mathbf{5 c}$, and $\mathbf{5 e}$ proved active, while their analogue $\beta$-amino alcohols ${ }^{12}$ showed no activity against $S$. aureus or E. coli. Table 4 depicts the lipophilicity

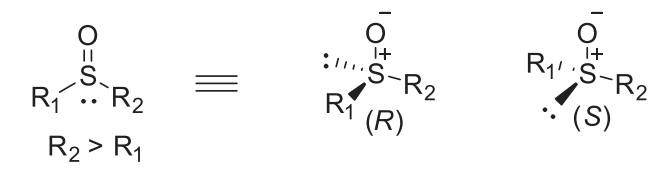

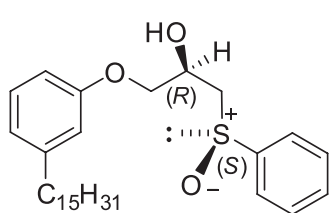

la

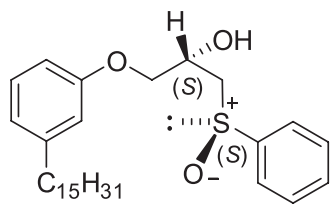

Ila<smiles>CCCCCCc1cccc(OC[C@H](O)[C@H](C)S(C)(C)c2ccccc2)c1</smiles>

Ib<smiles>C[13CH2]c1cccc(OC[C@H](O)[C@H](O)C[Sb](C)(=O)c2ccccc2)c1</smiles>

IIb
Figure 4. Possible stereoisomers of $\mathbf{6 a}$.

values (expressed as $\log \mathrm{P}$ ) of the $\beta$-hydroxy sulfides, sulfoxides, and sulfones investigated.

\section{Antioxidant assay}

Sulfur-bearing compounds (thiols, disulfides, sulfides) have antioxidant properties. Sulfides are among the antioxidants capable of inactivating hydroperoxides without generating radical species. Effectively reacting with peroxyl radicals, sulfur-containing antioxidants are widely used as antioxidant additives in oils and polymers. ${ }^{32}$ To evaluate the capacity of $\beta$-hydroxy sulfides $\mathbf{5 a - 5 j}$ to reduce free radicals, an antioxidant-activity test was performed based on $\mathrm{DPPH}^{\circ}$, a stable radical that imparts purple coloration to solutions. $\mathrm{DPPH}{ }^{*}$ reduction to a hydrazine is accompanied by a decrease in absorbance. ${ }^{33}$

As shown in Figure 5, compounds $\mathbf{5 a - 5 c}$ failed to reduce $\mathrm{DPPH}^{\circ}$ at any of the concentrations tested $(\mathrm{Q}=0 \%)$, while 
Table 4. Minimum inhibitory concentration (MIC) and $\log \mathrm{P}$ values for compounds tested against bacterial strains

\begin{tabular}{|c|c|c|c|c|c|}
\hline \multirow{2}{*}{ Compound } & \multirow{2}{*}{$\log \mathrm{P}^{\mathrm{a}}$} & \multicolumn{2}{|c|}{ S. aureus (ATCC 25923) } & \multicolumn{2}{|c|}{ E. coli (ATCC 25922) } \\
\hline & & $\mathrm{MIC} /\left(\mu \mathrm{g} \mathrm{mL}^{-1}\right)$ & $\mathrm{MIC} / \mu \mathrm{M}$ & $\mathrm{MIC} /\left(\mu \mathrm{g} \mathrm{mL}^{-1}\right)$ & $\mathrm{MIC} / \mu \mathrm{M}$ \\
\hline $5 a$ & 9.34 & 125 & 265.5 & NA & - \\
\hline $5 \mathbf{b}$ & 9.45 & NA & - & NA & - \\
\hline $5 c$ & 9.49 & 125 & 247.7 & NA & - \\
\hline $5 d$ & 9.49 & 125 & 247.7 & NA & - \\
\hline $5 e$ & 9.50 & 125 & 247.7 & NA & - \\
\hline $5 f$ & 9.52 & NA & - & NA & - \\
\hline $5 \mathrm{~g}$ & 9.53 & NA & - & NA & - \\
\hline $5 \mathrm{~h}$ & 9.38 & 125 & 255.7 & NA & - \\
\hline $5 i$ & 9.06 & 125 & 257.3 & NA & - \\
\hline $5 \mathbf{j}$ & 8.97 & 125 & 295.7 & NA & - \\
\hline 6a-6j & $8.11-9.11$ & NA & - & NA & - \\
\hline $7 \mathbf{a}, 7 \mathbf{c}-7 \mathbf{f}, 7 \mathbf{h}-7 \mathbf{j}$ & $8.28-9.24$ & NA & - & NA & - \\
\hline Gentamicin & & $\leq 4.68$ & $\leq 9.8$ & $\leq 4.68$ & $\leq 9.8$ \\
\hline
\end{tabular}

${ }^{2}$ Theoretical $\log$ P calculated using the Molinspiration algorithm. ${ }^{28}$ NA: not active at the tested concentrations.

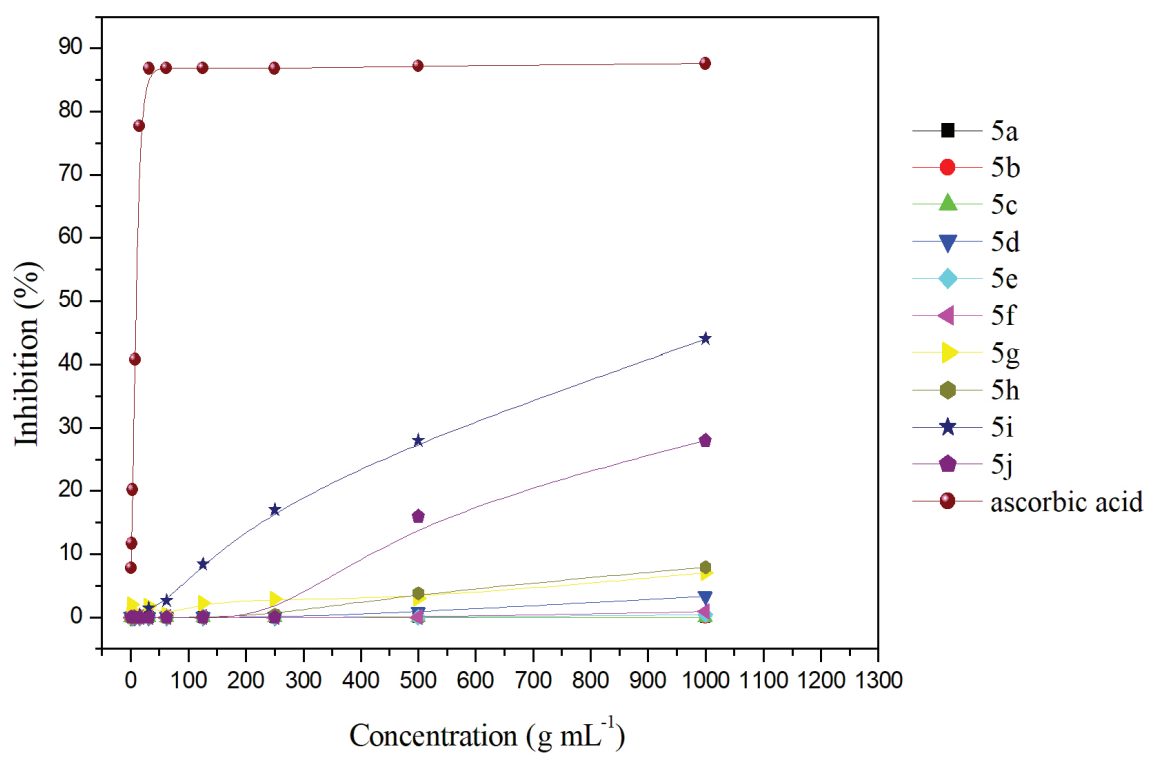

Figure 5. DPPH'-reducing activity displayed by $\beta$-hydroxy sulfides $\mathbf{5 a - 5 j}$ and standard ascorbic acid solution.

5d-5h exhibited very low scavenging activity, of 3.3, 0.4, $0.9,7.0$, and $7.9 \%$, respectively, at the highest concentration evaluated $\left(1 \mathrm{mg} \mathrm{mL}^{-1}\right)$. Compounds $\mathbf{5 i}$ and $\mathbf{5 j}$ proved moderately active: none of the concentrations investigated reduced $\mathrm{DPPH}^{*}$ by more than $50 \%$, precluding satisfactory estimation of half-maximal inhibitory concentration $\left(\mathrm{IC}_{50}\right)$ values. The standard ascorbic acid solution proved significantly more active than the compounds investigated.

Structural analysis of $\mathbf{5 i}$ and $\mathbf{5 j}$ provides support to make inferences about $\mathrm{DPPH}^{\cdot}$ reduction results. The aromatic ring of $\beta$-hydroxy sulfide $5 \mathbf{i}$ has an oxidable amine group para-positioned to the sulfur atom. Abstraction of a hydrogen radical from the amine group can produce, by resonance, a $\mathbf{5 i}$ stabilized radical, with one contributor taking the form of a stable quinone-type radical (Scheme 4).<smiles>N=C1C=CC(SCC(O)CO[Al])C=C1</smiles>

Scheme 4. Proposed reaction for DPPH• reduction by $\beta$-hydroxy sulfide $\mathbf{5 i}$.

Of the compounds evaluated, this sulfide exhibited the highest reducing activity.

In contrast to the other $\beta$-hydroxy sulfides investigated, $\mathbf{5 j}$ has an ethyl group, instead of an aromatic ring, attached to the sulfur atom. This structural feature may indicate, 


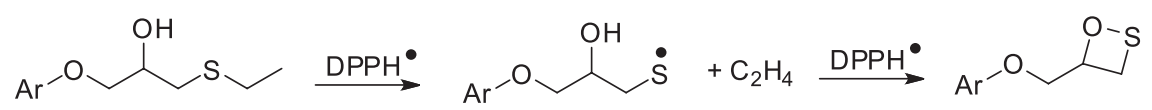

Scheme 5. Proposed radical $\beta$-elimination for $\beta$-hydroxy sulfide $\mathbf{5 j}$.

as proposed by Bridgewater and Sexton ${ }^{34}$ to explain the antioxidant potential of organic sulfides, the possibility of $\beta$-elimination of a hydrogen from the ethyl group by $\mathrm{DPPH}^{\circ}$, leading to rupture of the $\mathrm{C}-\mathrm{S}$ bond (Scheme 5). Also, the presence of a $\beta$-positioned hydroxyl may facilitate formation of a product of this mechanism if a second reaction with DPPH' takes place. In view of this background, the milder antioxidant activity displayed by other $\beta$-hydroxy sulfides can be attributed to the simultaneous absence of a $\beta$-hydrogen on one side of the $\mathrm{C}-\mathrm{S}$ bond and the presence of a hydroxyl on the opposite side of the sulfur atom.

\section{Conclusions}

Twenty eight novel organosulfur compounds ( $\beta$-hydroxy sulfides 5a-5j, $\beta$-hydroxy sulfoxides $\mathbf{6 a - 6} \mathbf{j}$, and $\beta$-hydroxy sulfones $\mathbf{7 a}, \mathbf{7} \mathbf{c}-\mathbf{7 f}, \mathbf{7 h}-\mathbf{7} \mathbf{j}$ ) were synthesized from a molecular combination of cardanol and glycerol, with excellent yields and employing more environmentally friendly processes. All the substances were evaluated for antibacterial activity against standard strains of $S$. aureus (Gram-positive) and E. coli (Gram-negative). MIC for $S$. aureus was highest for $\mathbf{5 a}, \mathbf{5 c}-\mathbf{5 e}$ and $\mathbf{5 h}-\mathbf{5 j}$. None of the tested substances proved active against E. coli. Compounds $\mathbf{5 a - 5 j}$ were also evaluated as antioxidant agents using the $\mathrm{DPPH}^{\bullet}$ method. Substances with lowest $\log \mathrm{P}$ values $(\mathbf{5 i}, \mathbf{5 j})$ exhibited highest radical-scavenging activities (44 and 28\%, respectively), while the remaining compounds proved inactive or minimally active (0.4-7.9\%). These findings are expected to play a crucial role in the development of novel antibacterial agents.

\section{Supplementary Information}

Supplementary data (NMR spectra) are available free of charge at http://jbcs.sbq.org.br as a PDF file.

\section{Acknowledgments}

The authors are grateful to the Universidade Federal de Mato Grosso do Sul, the Fundação de Apoio ao Desenvolvimento do Ensino, Ciência e Tecnologia do Estado de Mato Grosso do Sul (Fundect-MS, ProNEm grant 054/12), the Brazilian Council for Scientific and Technological Development (CNPq), the Coordenação de Aperfeiçoamento de Pessoal de Nível Superior, Brazil
(CAPES), and Kardol Indústria Química Ltda. for their support of our investigations in this field. S. C. also thanks Fundect-MS for the $\mathrm{PhD}$ grant awarded. This investigation was partly funded by CAPES (finance code 001).

\section{Author Contributions}

Suély Copini and Alisson Meza were responsible for the data curation, investigation, and writing original draft; Adilson Beatriz, Dênis P. de Lima and Ana C. Micheletti for the formal analysis, funding acquisition, conceptualization and writing review and editing; Adilson Beatriz was also responsible for the project administration, resources, validation and visualization; Roberto S. Gomes was responsible for the data curation and writing review and editing.

\section{References}

1. Kornprobst, J.-M.; Sallenave, C.; Barnathan, G.; Comp. Biochem. Physiol., Part B: Biochem. Mol. Biol. 1998, 119, 1.

2. Marakalala, M. B.; Mmutlane, E. M.; Kinfe, H. H.; Beilstein J. Org. Chem. 2018, 14, 1668.

3. Prinsep, M. R. In Studies in Natural Products Chemistry, vol. 28; Rahman, A.-U., ed.; Elsevier: Amsterdam, The Netherlands, 2003, p. 617-751.

4. Tehri, P.; Aegurula, B.; Peddinti, R. K.; Tetrahedron Lett. 2017, 58, 2062.

5. Foster, H. R.; Fuerst, E.; Branchett, W.; Lee, T. H.; Cousins, D. J.; Woszczek, C.; Sci. Rep. 2016, 6, 20461.

6. Takada, N.; Umemura, N.; Suenaga, K.; Uemura, D.; Tetrahedron Lett. 2001, 42, 3495.

7. de Castro, M. V.; Ióca, L. P.; Williams, D. E.; Costa, B. Z.; Mizuno, C. M.; Santos, M. F. C.; de Jesus, K.; Ferreira, É. L. F.; Seleghim, M. H. R.; Sette, L. D.; Pereira Filho, E. R.; Ferreira, A. G.; Gonçalves, N. S.; Santos, R. A.; Patrick, B. O.; Andersen, R. J.; Berlinck, R. G. S. J.; Nat. Prod. 2016, 79, 1668.

8. O'Connor, S. E.; Grosset, A.; Janiak, P.; Fundam. Clin. Pharmacol. 1999, 13, 145.

9. Mazzeto, S. E.; Lomonaco, D.; Mele, G.; Quim. Nova 2009, 32, 732 .

10. Beatriz, A.; Araújo, Y. J. K.; de Lima, D. P.; Quim. Nova 2011, 34, 306; Mota, C. J. A.; da Silva, C. X. A.; Gonçalves, V. L. C.; Quim. Nova 2009, 32, 639.

11. Braga, F. C.; Avvari, N. P.; Gomes, R. S.; Nascimento, V. A.; Oliveira, S. L.; Caires, A. R. L.; de Lima, D. P.; Beatriz, A.; Dyes Pigm. 2017, 141, 235. 
12. Manda, B. R.; Avvari, N. P.; Thatikonda, N. R.; Lacerda Jr., V.; Barbosa, L. R.; Santos, H.; Romão, W.; Pavan, F. R.; Ribeiro, C. M.; dos Santos, E. A.; Marques, M. R.; de Lima, D. P.; Micheletti, A. C.; Beatriz, A.; J. Braz. Chem. Soc. 2018, 29, 639.

13. Paiva, D. R.; de Lima, D. P.; Avvari, N. P.; Arruda, E. J.; Cabrini, I.; Marques, M. R.; Santos, E. A.; Biaggio, F. C.; Sangi, D. P.; Beatriz, A.; An. Acad. Bras. Cienc. 2017, 89, 373.

14. Ganesh, V.; Chandrasekaran, S.; Synthesis 2009, 19, 3267.

15. Lanke, S. R.; Bhanage, B. M.; Catal. Commun. 2013, 41, 29.

16. Ahmad, S.; Zahoor, A. F.; Naqvi, S. A. R.; Akash, M.; Mol. Diversity 2018, 22, 191.

17. Chandrasekhar, S.; Reddy, C. R.; Babu, B. N.; Chandrashekar, G.; Tetrahedron Lett. 2002, 43, 3801.

18. Fringuelli, F.; Pizzo, F.; Tortoioli, S.; Vaccaro, L.; Tetrahedron Lett. 2003, 44, 6785.

19. Fringuelli, F.; Pizzo, F.; Tortoioli, S.; Vaccaro, L.; J. Org. Chem. 2003, 68, 8248.

20. Su, W.; Chen, J. X.; Wu, H. Y.; Jin, C.; J. Org. Chem. 2007, 72, 4524.

21. Bandgar, B. P.; Patil, A. V.; Chavan, O. S.; Kamble, V. T.; Catal. Commun. 2007, 8, 1065.

22. Zhu, J.; Li, R.; Ge, Z.; Cheng, T.; Li, R.; Chin. J. Chem. 2009 , 27,791 .
23. Mukherjee, C.; Maiti, G. H.; Misra, A. K.; ARKIVOC 2008, xi, 46.

24. Sato, K.; Aoki, M.; Noyori, R.; Science 1998, 281, 1646.

25. Beatriz, A.; Lima, D. P.; Arruda, E. J.; Paiva, D. R.; Cossa, T. M.; BR pat. 102014030002-3 A2 2016.

26. Micheletti, A. C.; Handa, N. K.; Carvalho, N. C. P.; de Lima, D. P.; Beatriz, A.; Orbital: Electron. J. Chem. 2015, 7, 301.

27. Uriarte-Pueyo, I.; Calvo, M. I.; Food Chem. 2010, 120, 679.

28. http://www.molinspiration.com/cgi-bin/properties, accessed in June 2020.

29. Pinacho, R.; Cavero, R. Y.; Astiasarán, I.; Ansorena, D.; Calvo, M. I.; J. Funct. Foods 2015, 19, 49.

30. Kuete, V.; Planta Med. 2010, 76, 1479.

31. Rezende Jr., C. O.; Oliveira, L. A.; Oliveira, B. A.; Almeida, C. G.; Ferreira, B. S.; Le Hyaric, M.; Carvalho, G. S. L.; Lourenço, M. C. S.; Batista, M.; Marchini, F. K.; Silva, V. L.; Diniz, C. G.; Almeida, M. V.; Chem. Biol. Drug Des. 2015, 86, 344.

32. Smolyaninov, I. V.; Pitikova, O. V.; Rychagova, E. S.; Korchagina, E. O.; Poddel'sky, A. I.; Smolyaninova, S. A.; Berberova, N. T.; Russ. Chem. Bull. 2016, 65, 2861.

33. Blois, M. S.; Nature 1958, 181, 1199.

34. Bridgewater, A. J.; Sexton, M. D.; J. Chem. Soc., Perkin Trans. 2 1978, 530. 